\title{
Realización electrónica de sistemas caóticos: Parte 1, Analógicos cuadráticos
}

\author{
Francisco Antonio Rodríguez Cruz, César de Jesús Chacón Rendón, \\ Angel Rodriguez-Liñan \\ Universidad Autónoma de Nuevo León, Facultad de Ingeniería Mecánica y Eléctrica \\ franciscoantoniordz@hotmail.com, ces.chre98@gmail.com, angel.rodriguezln@uanl.edu.mx
}

\section{RESUMEN}

Algunas aplicaciones de sistemas caóticos requieren su implementación mediante electrónica analógica o digital. En esta primera parte, se resume la realización electrónica de algunos sistemas caóticos más conocidos con no linealidades cuadráticas, mediante circuitos con multiplicadores y amplificadores operacionales. Se demuestra la equivalencia de sus variables y componentes electrónicos con las variables y parámetros normalizados de los modelos matemáticos caóticos. Además, se compara el comportamiento de cada circuito con simulaciones de sus modelos matemáticos establecidos. En las siguientes partes de este trabajo, se mostrará la realización electrónica de sistemas caóticos lineales por tramos e implementación en dispositivos digitales.

\section{PALABRAS CLAVE}

Sistemas caóticos, Computación analógica, Amplificadores operacionales, No linealidad cuadrática.

\section{ABSTRACT}

Some applications of chaotic systems require their implementation through analog or digital electronics. In this first part, the electronic realization of some well-known chaotic systems with quadratic nonlinearities is summarized, by means of circuits with multipliers and operational amplifiers. The equivalence of its variables and electronic components with the normalized variables and parameters of chaotic mathematical models is demonstrated. In addition, the behavior of each circuit is compared to simulations of their mathematical models. In the following parts of this work, the electronic realization of piecewise linear chaotic systems and implementation in digital devices will be shown.

\section{KEYWORDS}

Chaotic systems, Analog computation, Operational amplifiers, Quadratic nonlinearity.

\section{INTRODUCCIÓN}

Un sistema caótico es un sistema dinámico con varias características, entre las principales está su sensibilidad a las condiciones iniciales, ya que, al variarlas, un sistema caótico presenta un resultado completamente diferente al obtenido con las condiciones iniciales originales, pero siempre trazando un patrón característico. Los sistemas caóticos continuos de orden entero y dimensión finita pueden modelarse mediante ecuaciones diferenciales ordinarias y los sistemas caóticos discretos pueden describirse con ecuaciones en diferencias iterativas. 
Usualmente el comportamiento de sus variables con respecto al tiempo parece errático, además de extremadamente difícil de predecir después de cierto horizonte de tiempo. Por ejemplo, es muy difícil predecir (o imposible) el comportamiento atmosférico para horizontes temporales mayores a varios días o semanas ${ }^{1}$. Sin embargo, si se estudia el comportamiento de dichas variables en el espacio de fase en lugar de en el espacio temporal, el comportamiento de las variables luce más repetitivo e interpretable, describiendo una trayectoria espacial característica, a la que se denomina atractor caótico. Los sistemas, señales y atractores caóticos han sido estudiados en diversos fenómenos de la naturaleza y sociedad, así como para aplicaciones en muchas disciplinas de la ingeniería. Por ejemplo, en los fenómenos meteorológicos, en biología en crecimiento poblacional, en física para el estudio de sincronización, para el estudio de la turbulencia en fluidos, se presentan en sistemas mecánicos y circuitos electrónicos, se proponen en sistemas de comunicación para el cifrado de señales, entre otros casos donde se estudian los atractores caóticos ${ }^{2-8}$. Algunas de estas aplicaciones requieren la implementación de los sistemas caóticos mediante circuitería electrónica analógica y otras de forma digital.

\section{ANTECEDENTES}

Desde hace algunas décadas, se han creado circuitos analógicos que reproducen algunos atractores de sistemas caóticos algebraicamente simples, como el sistema de Rössler ${ }^{9,10}$, que es un conjunto de tres ecuaciones diferenciales donde sólo se presenta una no linealidad cuadrática (producto multiplicativo). También se ha implementado electrónicamente el sistema de Lorenz, que es un sistema de tres ecuaciones diferenciales con sólo dos productos multiplicativos que, aunque surgió a partir del fenómeno de convección en la atmósfera, es aplicado en telecomunicaciones ${ }^{2}$ y estudiado en sincronización de caos ${ }^{11}$. De este sistema se derivan otros circuitos similares, como el de Chen y el de L $\ddot{u ̈}^{12-14}$. Los sistemas caóticos de Malasoma son de las estructuras más simples, que sólo contienen cada uno un término cuadrático o producto multiplicativo ${ }^{15}$. Aunque parece fácil la implementación o realización de sistemas caóticos con circuitería analógica, se requiere proponer mallas y ramas de componentes que permitan reconstruir cada una de las ecuaciones diferenciales que describen a los sistemas caóticos, así como elegir adecuadamente el valor comercial de los componentes electrónicos; ya que, por su naturaleza caótica, estos circuitos son muy sensibles a variaciones paramétricas y estructura en las ecuaciones de su dinámica. Por ello, es conveniente conocer el modelo matemático del circuito y la relación de sus variables con las ecuaciones diferenciales del sistema caótico, para hacer análisis, aplicaciones y control de sus patrones de oscilación basados en su dinámica. Sin embargo, en muchos casos reportados en la literatura no se explica con detalle cómo se construye cada una de las ecuaciones diferenciales de los circuitos, ni cómo son equivalentes a los sistemas caóticos adimensionales reportados. Además, no suelen explicar cómo seleccionar el valor de los componentes electrónicos que les permitan seguir siendo equivalentes al sistema caótico.

\section{CONTRIBUCIÓN}

En este trabajo se resume la realización de varios de los sistemas caóticos más conocidos, mediante circuitos electrónicos analógicos y digitales, que permiten generar sus atractores característicos. Además, se demuestra la equivalencia entre sus variables y componentes electrónicos con las variables y parámetros adimensionales de los modelos matemáticos caóticos. También, se propone un circuito unificado para los sistemas de Lorenz, Chen y Lü. Para mayor detalle, este trabajo se ha organizado en tres partes: En la primera, se ilustra la realización y modelado de varios sistemas caóticos más conocidos con no linealidades cuadráticas, utilizando multiplicadores y amplificadores operacionales (op-amp, por sus siglas en inglés). En la segunda parte, se mostrará la realización de sistemas caóticos mediante circuitos analógicos lineales por tramos, así como el mapeo entre magnitudes eléctricas y variables de los modelos caóticos adimensionales. En la tercera parte, se aplica discretización en el tiempo a sistemas caóticos, para su implementación digital en la plataforma Arduino y adquisición de datos. En cada parte, se ilustran resultados mediante simulaciones e implementación de circuitos. 


\section{REALIZACIÓN CON CIRCUITOS ANALÓGICOS}

En esta sección, se resume la realización electrónica de algunos sistemas caóticos utilizando resistores, capacitores, multiplicadores de voltaje y op-amp en las configuraciones conocidas como amplificador inversor, amplificador no inversor, sumador inversor, restador inversor e integrador ${ }^{16}$. También se presenta el mapeo entre las magnitudes eléctricas de dichos componentes y las variables de sus modelos caóticos adimensionales.

\section{Sistemas de Lorenz, Lü y Chen}

Un sistema caótico unificado ${ }^{14}$ es descrito por

$$
\left\{\begin{array}{l}
\frac{d x_{1}}{d \tau}=(25 \gamma+10)\left(x_{2}-x_{1}\right) \\
\frac{d x_{2}}{d \tau}=(28-35 \gamma) x_{1}+(29 \gamma-1) x_{2}-x_{1} x_{3} \\
\frac{d x_{3}}{d \tau}=x_{1} x_{2}-\frac{(\gamma+8)}{3} x_{3}
\end{array}\right.
$$

donde $\tau$ es una variable normalizada de tiempo, $x_{1}, x_{2}$ y $x_{3}$ son las variables de estado y $\gamma \in[0,1]$. Cuando $\gamma \in[0,0.8)$, el sistema (1) coincide con el sistema caótico de Lorenz, que se representa típicamente ${ }^{1}$ como

$$
\left\{\begin{array}{l}
\frac{d x_{1}}{d \tau}=\sigma\left(x_{2}-x_{1}\right) \\
\frac{d x_{2}}{d \tau}=\rho x_{1}-x_{2}-x_{1} x_{3} \\
\frac{d x_{3}}{d \tau}=x_{1} x_{2}-b x_{3}
\end{array}\right.
$$

con $\sigma, \rho$ y $b$ como parámetros constantes. Si $\gamma=0.8$, el sistema (1) coincide con el sistema caótico de Lü, que se representa típicamente ${ }^{17}$ como

$$
\left\{\begin{array}{l}
\frac{d x_{1}}{d \tau}=a\left(x_{2}-x_{1}\right) \\
\frac{d x_{2}}{d \tau}=c x_{2}-x_{1} x_{3} \\
\frac{d x_{3}}{d \tau}=x_{1} x_{2}-b x_{3}
\end{array}\right.
$$

con $a, c$ y $b$ como parámetros constantes. Si $\gamma \in(0.8,1]$, el sistema (1) coincide con el sistema caótico de Chen, que se representa típicamente ${ }^{18}$ como

$$
\left\{\begin{array}{l}
\frac{d x_{1}}{d \tau}=a\left(x_{2}-x_{1}\right) \\
\frac{d x_{2}}{d \tau}=(c-a) x_{1}+c x_{2}-x_{1} x_{3} \\
\frac{d x_{3}}{d \tau}=x_{1} x_{2}-b x_{3}
\end{array}\right.
$$

con $a, c$ y $b$ como parámetros constantes. Se propone el circuito de la figura 1 como una misma realización electrónica de los sistemas (2), (3) y (4), donde $R_{1}, R_{2}, \ldots, R_{14}$ son resistencias, $C_{1}, C_{2}$ y $C_{3}$ son capacitancias, $A_{1}$ y $A_{2}$ son ganancias de los multiplicadores de voltaje, $S_{0}$ y $S_{1}$ son selectores, $v_{1}, v_{2}$ y $v_{3}$ son los voltajes de salida de los op-amp U1B, U2B y U3B, respectivamente. 


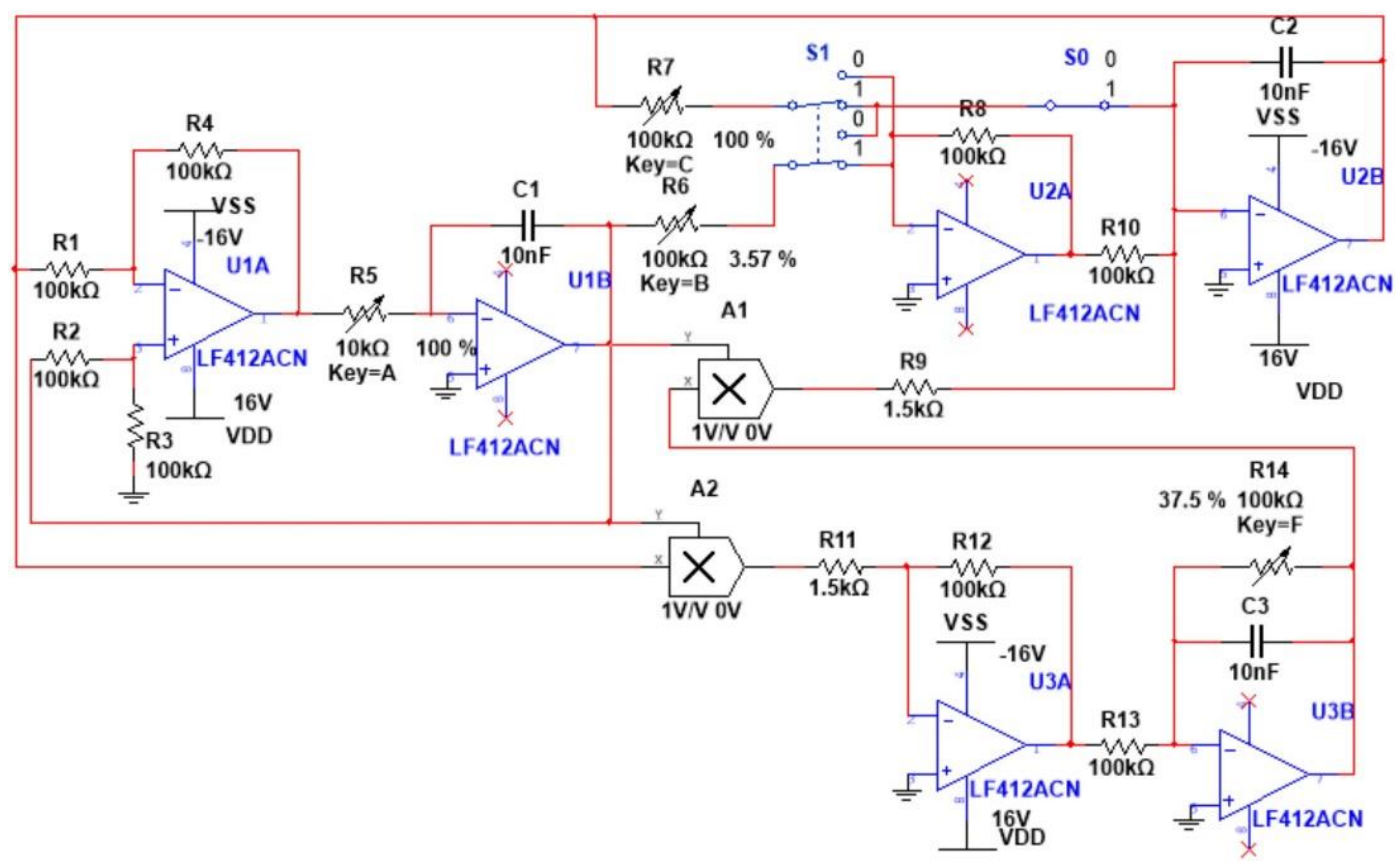

Fig. 1. Realización electrónica unificada de los sistemas Lorenz, Lü y Chen.

Para demostrar la equivalencia del circuito de la figura 1 con los sistemas (2), (3) y (4), se realiza el siguiente análisis:

Se inicia con el op-amp U1A, si $R_{1}=R_{2} y R_{3}=R_{4}$, entonces su voltaje de salida es

$$
V_{1 A}=\left(\frac{R_{3}}{R_{2}+R_{3}}\right)\left(1+\frac{R_{4}}{R_{1}}\right) v_{1}-\left(\frac{R_{4}}{R_{1}}\right) v_{2}=\left(\frac{R_{4}}{R_{1}}\right)\left(v_{1}-v_{2}\right)
$$

Para el op-amp U1B, su voltaje de salida es

$$
v_{1}=-\frac{1}{R_{5} C_{1}} \int V_{1 A} d t=\frac{1}{R_{1} C_{1}} \int\left(\frac{R_{4}}{R_{5}}\right)\left(v_{2}-v_{1}\right) d t
$$

donde $t$ es la variable de tiempo. Para el op-amp U2A, dependiendo del estado del selector $S_{1} \in\{0,1\}$, su voltaje de salida es

$$
V_{2 A}=-S_{1}\left(\frac{R_{8}}{R_{6}}\right) v_{1}-\left(1-S_{1}\right)\left(\frac{R_{8}}{R_{7}}\right) v_{2}
$$

Para el op-amp U2B, si $R_{8}=R_{10}$, dependiendo del estado de los selectores $S_{0} \in\{0,1\}$ y $S_{1} \in\{0,1\}$, se cumple

$$
\begin{gathered}
\frac{V_{2 A}}{R_{10}}+\frac{A_{1} v_{1} v_{3}}{R_{9}}+S_{0}\left(S_{1} \frac{v_{2}}{R_{7}}+\left(1-S_{1}\right) \frac{v_{1}}{R_{6}}\right)+C_{2} \frac{d v_{2}}{d t}=0 \\
\frac{d v_{2}}{d t}=\frac{1}{C_{2}}\left(-\frac{V_{2 A}}{R_{10}}-\frac{A_{1} v_{1} v_{3}}{R_{9}}-S_{0}\left(S_{1} \frac{v_{2}}{R_{7}}+\left(1-S_{1}\right) \frac{v_{1}}{R_{6}}\right)\right) \\
\frac{d v_{2}}{d t}=\frac{1}{C_{2}}\left(S_{1}\left(\frac{R_{8}}{R_{6} R_{10}}\right) v_{1}+\left(1-S_{1}\right)\left(\frac{R_{8}}{R_{7} R_{10}}\right) v-\frac{A_{1} v_{1} v_{3}}{R_{9}}-S_{0} S_{1} \frac{v_{2}}{R_{7}}-S_{0}\left(1-S_{1}\right) \frac{v_{1}}{R_{6}}\right) \\
\frac{d v_{2}}{d t}=\frac{1}{C_{2}}\left(\left[S_{1}-S_{0}\left(1-S_{1}\right)\right]\left(\frac{1}{R_{6}}\right) v_{1}+\left[\left(1-S_{1}\right)-S_{0} S_{1}\right]\left(\frac{1}{R_{7}}\right) v_{2}-\frac{A_{1} v_{1} v_{3}}{R_{9}}\right)
\end{gathered}
$$

Para el op-amp U3A, el voltaje de salida es

$$
V_{3 A}=-\left(\frac{R_{12}}{R_{11}}\right) A_{2} v_{1} v_{2}
$$


Para el op-amp U3B, se cumple

$$
\begin{gathered}
\frac{V_{3 A}}{R_{13}}+\frac{v_{3}}{R_{14}}+C_{3} \frac{d v_{3}}{d t}=0 \\
\frac{d v_{3}}{d t}=\frac{1}{C_{3}}\left(-\frac{V_{3 A}}{R_{13}}-\frac{v_{3}}{R_{14}}\right)=\frac{1}{C_{3}}\left(\left(\frac{R_{12}}{R_{11} R_{13}}\right) A_{2} v_{1} v_{2}-\frac{v_{3}}{R_{14}}\right)
\end{gathered}
$$

Por lo tanto, partiendo de las ecuaciones (5), (6) y (7), el circuito electrónico de la figura 1 puede modelarse por

$$
\left\{\begin{array}{l}
\frac{d v_{1}}{d t}=\frac{1}{R_{1} C_{1}}\left(\frac{R_{4}}{R_{5}}\right)\left(v_{2}-v_{1}\right) \\
\frac{d v_{2}}{d t}=\frac{1}{R_{10} C_{2}}\left(\left[S_{1}-S_{0}\left(1-S_{1}\right)\right]\left(\frac{R_{10}}{R_{6}}\right) v_{1}+\left[\left(1-S_{1}\right)-S_{0} S_{1}\right]\left(\frac{R_{10}}{R_{7}}\right) v_{2}-\left(\frac{A_{1} R_{10}}{R_{9}}\right) v_{1} v_{3}\right) \\
\frac{d v_{3}}{d t}=\frac{1}{R_{13} C_{3}}\left(\left(\frac{A_{2} R_{12}}{R_{11}}\right) v_{1} v_{2}-\left(\frac{R_{13}}{R_{14}}\right) v_{3}\right)
\end{array}\right.
$$

El modelo (8) es equivalente al sistema caótico de Lorenz adimensional (2), bajo las condiciones y mapeos del tiempo, estados y parámetros dados por

$\tau=\frac{1}{R_{1} C_{1}} t, \frac{v_{1}}{x_{1}}=\frac{v_{2}}{x_{2}}=\frac{v_{3}}{x_{3}}=M, M=\frac{R_{9}}{A_{1} R_{10}}=\frac{R_{11}}{A_{2} R_{12}}, \sigma=\frac{R_{4}}{R_{5}}, \rho=\frac{R_{10}}{R_{6}}, \quad b=\frac{R_{13}}{R_{14}}, C_{1}=C_{2}=C_{3}, R_{1}=R_{2}=R_{7}=$ $R_{8}=R_{10}=R_{13}, R_{3}=R_{4}, S_{0}=1$ y $S_{1}=1$.

El modelo (8) es equivalente al sistema caótico de Lü adimensional (3), bajo las condiciones y mapeos del tiempo, estados y parámetros dados por $\tau=\frac{1}{R_{1} C_{1}} t, \frac{v_{1}}{x_{1}}=\frac{v_{2}}{x_{2}}=\frac{v_{3}}{x_{3}}=M, M=\frac{R_{9}}{A_{1} R_{10}}=\frac{R_{11}}{A_{2} R_{12}}, a=\frac{R_{4}}{R_{5}}, b=\frac{R_{13}}{R_{14}}, \quad c=\frac{R_{10}}{R_{7}}$, $C_{1}=C_{2}=C_{3}, R_{1}=R_{2}=R_{8}=R_{10}=R_{13}, R_{3}=R_{4}$, el valor de $R_{6}$ no importa, $S_{0}=0$ y $S_{1}=0$.

El modelo (8) es equivalente al sistema caótico de Chen adimensional (4), bajo las condiciones y mapeos del tiempo, estados y parámetros dados por $\tau=\frac{1}{R_{1} C_{1}} t, \frac{v_{1}}{x_{1}}=\frac{v_{2}}{x_{2}}=\frac{v_{3}}{x_{3}}=M, M=\frac{R_{9}}{A_{1} R_{10}}=\frac{R_{11}}{A_{2} R_{12}}, a=\frac{R_{4}}{R_{5}}, b=\frac{R_{13}}{R_{14}}, c=$ $\frac{R_{10}}{R_{7}},(c-a)=\frac{R_{10}}{R_{6}}, C_{1}=C_{2}=C_{3}, R_{1}=R_{2}=R_{8}=R_{10}=R_{13}, R_{3}=R_{4}, S_{0}=1$ y $S_{1}=0$.

\section{Circuito Rössler}

El sistema caótico Rössler ${ }^{9}$ está representado por

$$
\left\{\begin{array}{l}
\frac{d x_{1}}{d \tau}=-x_{2}-x_{3} \\
\frac{d x_{2}}{d \tau}=x_{1}+a x_{2} \\
\frac{d x_{3}}{d \tau}=b+x_{3}\left(x_{1}-c\right)
\end{array}\right.
$$

donde $x_{1}, x_{2}$ y $x_{3}$ son las variables de estado, $\tau$ es una variable normalizada de tiempo, $a, b$ y $c$ son parámetros constantes. El sistema (9) tiene una realización electrónica por el circuito de la figura 2, donde $R_{1}, R_{2}, \ldots, R_{12}$ son resistencias, $R_{13}$ es un potenciómetro, $C_{1}, C_{2}$ y $C_{3}$ son capacitancias, $A_{1}$ es la ganancia del multiplicador, $v_{1}, v_{2} \mathrm{y}$ $v_{3}$ son los voltajes de salida de los op-amp U3B, U1B y U2B, respectivamente. 


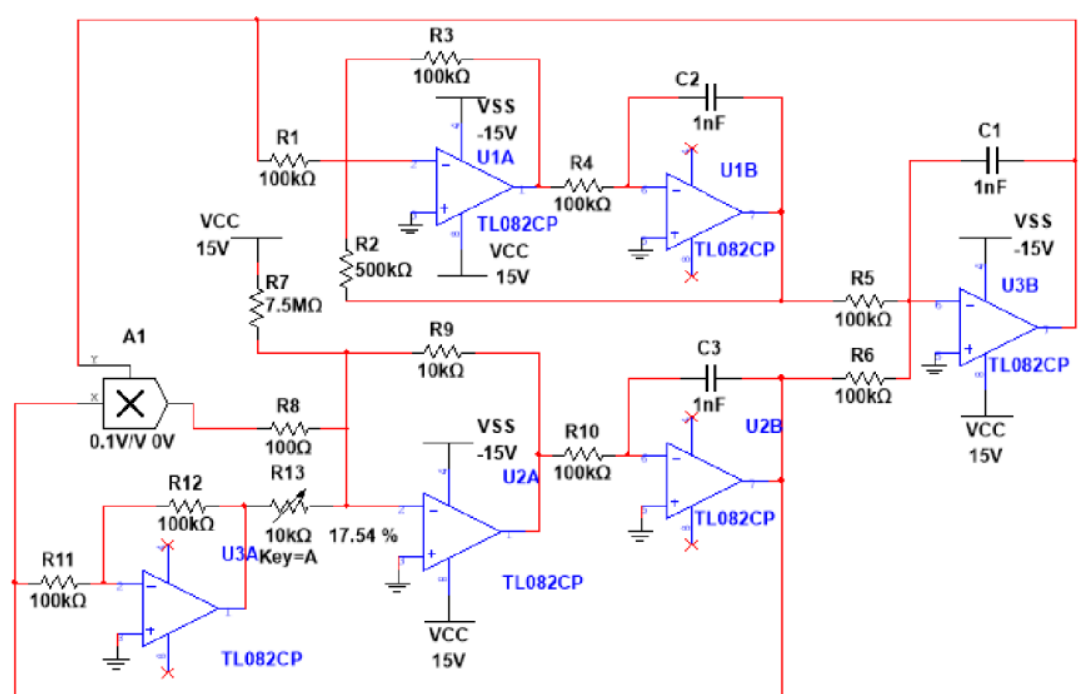

Fig. 2. Diagrama esquemático para la realización electrónica del sistema Rössler.

Para demostrar la equivalencia del circuito de la figura 2 con el sistema (9) se realiza el siguiente análisis: Analizando primero el op-amp U3B, su voltaje de salida es

$$
v_{1}=-\frac{1}{C_{1}} \int\left(\frac{v_{2}}{R_{5}}+\frac{v_{3}}{R_{6}}\right) d t
$$

donde $t$ es la variable de tiempo. Continuando con el op-amp U1A, su voltaje de salida es

Para el op-amp U1B, su voltaje de salida es

$$
V_{1 \mathrm{~A}}=-\frac{R_{3}}{R_{1}} v_{1}-\frac{R_{3}}{R_{2}} v_{2}
$$

$$
v_{2}=\frac{1}{R_{4} C_{2}} \int\left(\frac{R_{3}}{R_{1}} v_{1}+\frac{R_{3}}{R_{2}} v_{2}\right) d t
$$

Para el op-amp U3A, su voltaje de salida es

$$
V_{3 A}=-\frac{R_{12}}{R_{11}} v_{3}
$$

Para el op-amp U2A, su voltaje de salida es

$$
V_{2 A}=-\frac{R_{9}}{R_{7}} V_{\mathrm{CC}}-\frac{A_{1} R_{9}}{R_{8}} v_{1} v_{3}+\frac{R_{9} R_{12}}{R_{13} R_{11}} v_{3}
$$

Finalmente, el voltaje de salida del op-amp U2B es

$$
v_{3}=\frac{1}{R_{10} C_{3}} \int\left(\frac{R_{9}}{R_{7}} V_{\mathrm{CC}}+\frac{A_{1} R_{9}}{R_{8}} v_{1} v_{3}-\frac{R_{9} R_{12}}{R_{13} R_{11}} v_{3}\right) d t
$$

Por lo tanto, partiendo de las ecuaciones (10), (11) y (12), si $R_{1}=R_{3}, R_{5}=R_{6}$, el circuito electrónico de la figura 1 puede ser modelado por

$$
\left\{\begin{array}{l}
\frac{d v_{1}}{d t}=\frac{1}{R_{5} C_{1}}\left(-v_{2}-v_{3}\right) \\
\frac{d v_{2}}{d t}=\frac{1}{R_{4} C_{2}}\left(v_{1}+\frac{R_{3}}{R_{2}} v_{2}\right) \\
\frac{d v_{3}}{d t}=\frac{1}{R_{10} C_{3}}\left[\frac{R_{9}}{R_{7}} V_{\mathrm{CC}}+v_{3}\left(\frac{A_{1} R_{9}}{R_{8}} v_{1}-\frac{R_{9} R_{12}}{R_{13} R_{11}}\right)\right]
\end{array}\right.
$$


El modelo (13) es equivalente al sistema caótico de Rössler adimensional (9), bajo las condiciones y mapeos del tiempo, estados y parámetros dados por $\tau=\frac{1}{R_{5} C_{1}} t, \frac{v_{1}}{x_{1}}=\frac{v_{2}}{x_{2}}=\frac{v_{3}}{x_{3}}=M, M=\frac{R_{8}}{A_{1} R_{9}}, a=\frac{R_{3}}{R_{2}}, b=\frac{R_{9} V_{\mathrm{CC}}}{R_{7} M}, c=\frac{R_{9} R_{12}}{R_{13} R_{11}}$, $R_{1}=R_{3}, R_{4}=R_{5}=R_{6}=R_{10}, C_{1}=C_{2}=C_{3}$.

\section{Circuito de Liu-Chen}

El sistema de Liu-Chen ${ }^{19}$ se representa por

$$
\left\{\begin{array}{l}
\frac{d x_{1}}{d \tau}=a x_{1}-x_{2} x_{3} \\
\frac{d x_{2}}{d \tau}=-b x_{2}+x_{1} x_{3} \\
\frac{d x_{3}}{d \tau}=-c x_{3}+x_{1} x_{2}
\end{array}\right.
$$

donde $x_{1}, x_{2}$ y $x_{3}$ son las variables de estado, $\tau$ es una variable normalizada de tiempo, $a, b$ y $c$ son parámetros constantes. El sistema (14) tiene una realización electrónica por el circuito de la figura 3 , donde $R_{1}, R_{2}, \ldots, R_{12}$ son resistencias; $C_{1}, C_{2}$ y $C_{3}$, capacitancias; $A_{1}, A_{2}$ y $A_{3}$ son ganancias de los multiplicadores de voltaje; $v_{1}, v_{2} \mathrm{y}$ $v_{3}$ son los voltajes de salida de los op-amps U1B, U1C y U1D, respectivamente. $V_{D D}$ y $V_{S S}$ son los voltajes de alimentación de los amplificadores operacionales U1A, U1B, U1C, U1D, U2A y U2B.

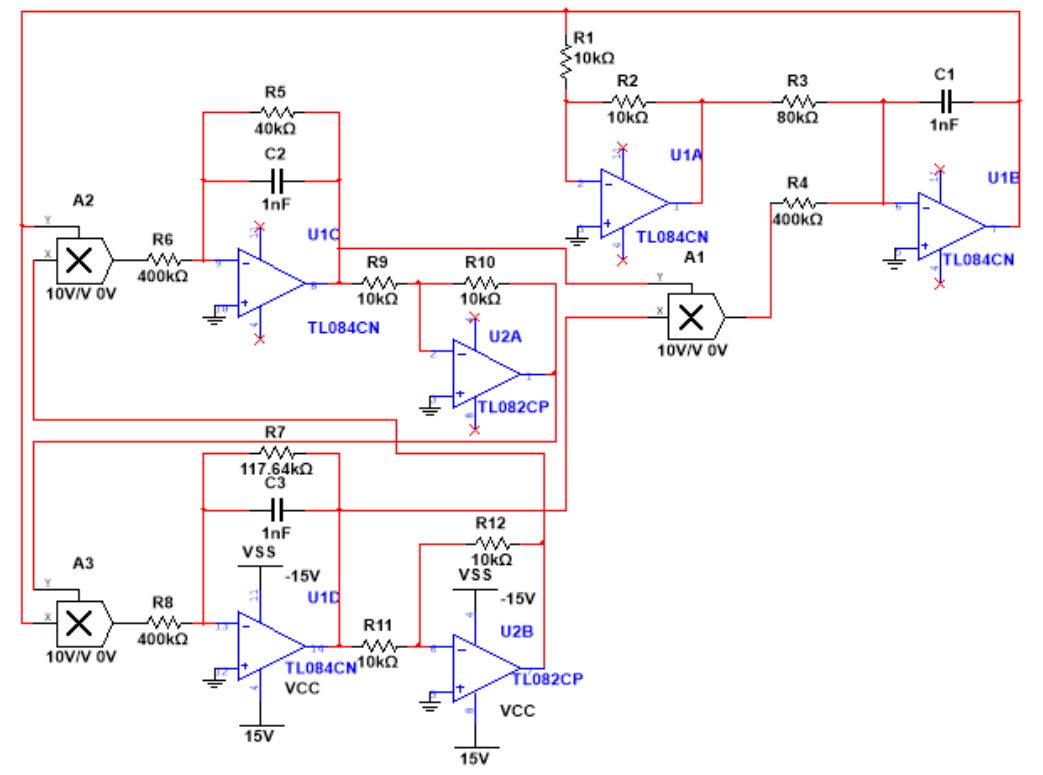

Fig. 3. Diagrama esquemático para la realización electrónica del sistema Liu-Chen.

Para demostrar la equivalencia del circuito de la figura 3 con el sistema (14) se realiza el siguiente análisis:

El voltaje de salida del op-amp U1A es

El voltaje de salida del op-amp U1B:

$$
V_{1 A}=-\frac{R_{2}}{R_{1}} v_{1}
$$

$$
v_{1}=\frac{1}{C_{1}} \int\left(-\frac{V_{1 A}}{R_{3}}-\frac{A_{1} v_{2} v_{3}}{R_{4}}\right) d t=\frac{1}{C_{1}} \int\left(\frac{R_{2} v_{1}}{R_{1} R_{3}}-\frac{A_{1} v_{2} v_{3}}{R_{4}}\right) d t
$$

donde $t$ es la variable de tiempo. El voltaje de salida del op-amp U2B es

$$
V_{2 B}=-\frac{R_{12}}{R_{11}} v_{3}
$$


Las corrientes en el nodo de la entrada inversora del op-amp U1C son:

$$
\frac{A_{2} v_{1} V_{2 B}}{R_{6}}+\frac{v_{2}}{R_{5}}+C_{2} \frac{d v_{2}}{d t}=-\frac{A_{2} R_{12} v_{1} v_{3}}{R_{6} R_{11}}+\frac{v_{2}}{R_{5}}+C_{2} \frac{d v_{2}}{d t}=0
$$

El voltaje de salida del op-amp U1C es

$$
v_{2}=\frac{1}{C_{2}} \int\left(-\frac{v_{2}}{R_{5}}+\frac{A_{2} R_{12} v_{1} v_{3}}{R_{6} R_{11}}\right) d t
$$

El voltaje de salida del op-amp U2A es

$$
V_{2 A}=-\frac{R_{10}}{R_{9}} v_{2}
$$

Las corrientes en el nodo de la entrada inversora del op-amp U1D es

$$
\frac{A_{3} v_{1} V_{2 A}}{R_{8}}+\frac{v_{3}}{R_{7}}+C_{3} \frac{d v_{3}}{d t}=-\frac{A_{3} R_{10} v_{1} v_{2}}{R_{8} R_{9}}+\frac{v_{3}}{R_{7}}+C_{3} \frac{d v_{3}}{d t}=0
$$

El voltaje de salida del op-amp U1D es

$$
v_{3}=\frac{1}{C_{3}} \int\left(-\frac{v_{3}}{R_{7}}+\frac{A_{3} R_{10} v_{1} v_{2}}{R_{8} R_{9}}\right) d t
$$

Por lo tanto, las ecuaciones (15), (16) y (17) pueden rescribirse como el modelo

$$
\left\{\begin{array}{l}
\frac{d v_{1}}{d t}=\frac{1}{R_{4} C_{1}}\left(\frac{R_{2} R_{4} v_{1}}{R_{1} R_{3}}-A_{1} v_{2} v_{3}\right) \\
\frac{d v_{2}}{d t}=\frac{1}{R_{6} C_{2}}\left(-\frac{R_{6} v_{2}}{R_{5}}+\frac{A_{2} R_{12} v_{1} v_{3}}{R_{11}}\right) \\
\frac{d v_{3}}{d t}=\frac{1}{R_{8} C_{3}}\left(-\frac{R_{8} v_{3}}{R_{7}}+\frac{A_{3} R_{10} v_{1} v_{2}}{R_{9}}\right)
\end{array}\right.
$$

El modelo (18) es equivalente al sistema caótico de Liu-Chen adimensional (14), bajo las condiciones y mapeos del tiempo, estados y parámetros dados por

$\tau=\frac{1}{R_{4} C_{1}} t, \frac{v_{1}}{x_{1}}=\frac{v_{2}}{x_{2}}=\frac{v_{3}}{x_{3}}=M, M=\frac{1}{A_{1}}=\frac{R_{11}}{A_{2} R_{12}}=\frac{R_{9}}{A_{3} R_{10}}, a=\frac{R_{2} R_{4}}{R_{1} R_{3}}, b=\frac{R_{6}}{R_{5}}, c=\frac{R_{8}}{R_{7}}, R_{4}=R_{6}=R_{8}, C_{1}=C_{2}=C_{3}$.

\section{Circuito de Malasoma}

J.M. Malasoma ${ }^{15}$ encontró una familia de sistemas caóticos algebraicamente muy simples y equivalentes entre sí, con una sola no linealidad cuadrática cada uno.

Uno de esos sistemas es el siguiente:

$$
\left\{\begin{array}{l}
\frac{d x_{1}}{d \tau}=x_{2} \\
\frac{d x_{2}}{d \tau}=x_{3} \\
\frac{d x_{3}}{d \tau}=-\alpha x_{3}-x_{1}+x_{1} x_{2}
\end{array}\right.
$$

donde $x_{1}, x_{2}$ y $x_{3}$ son las variables de estado, $\tau$ es una variable normalizada de tiempo, $\alpha$ es un parámetro constante. El sistema (19) tiene una realización electrónica por el circuito de la figura 4, donde $R_{1}, R_{2}, \ldots, R_{7}$ son resistencias; $C_{1}, C_{2}$ y $C_{3}$ son capacitancias, $A_{1}$ es la ganancia del multiplicador; $v_{1}, v_{2}$ y $v_{3}$ son los voltajes de salida de los opamp U1A, U1B y U1C, respectivamente. $V_{1}$ y $V_{2}$ son los voltajes de alimentación de los amplificadores operacionales U1A, U1B, U1C y U1D. 


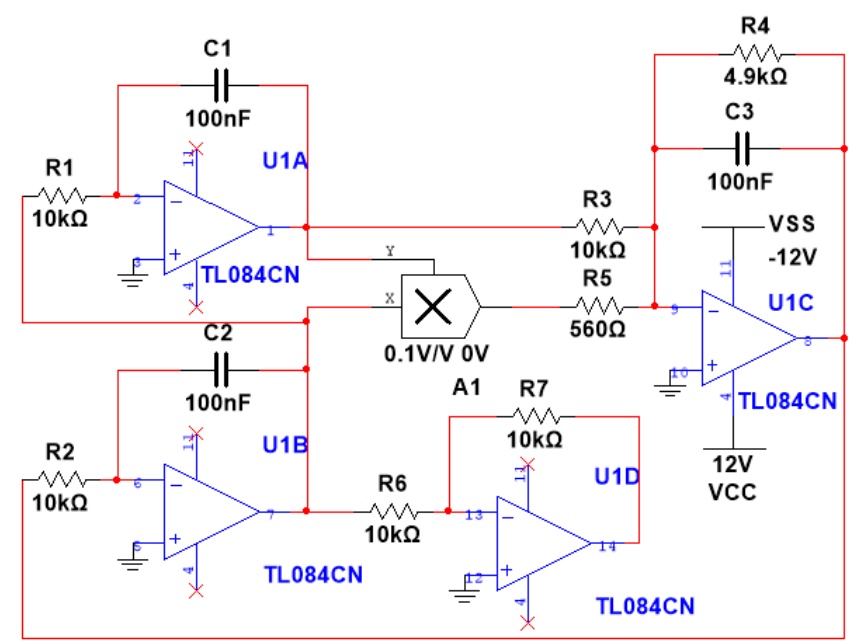

Fig. 4. Diagrama esquemático de una realización electrónica del sistema de Malasoma.

Para demostrar la equivalencia del circuito de la figura 4 con el sistema (19) se realiza el siguiente análisis: El voltaje de salida del op-amp U1B es

Si $R_{6}=R_{7}$, el voltaje de salida del op-amp U1D es

$$
V_{1 B}=-\frac{1}{C_{2}} \int\left(\frac{1}{R_{2}} v_{3}\right) d t
$$

$$
v_{2}=-\frac{R_{7}}{R_{6}} V_{1 B}=\frac{1}{C_{2}} \int\left(\frac{1}{R_{2}} v_{3}\right) d t
$$

El voltaje de salida del op-amp U1A es

$$
v_{1}=\frac{1}{C_{1}} \int\left(-\frac{1}{R_{1}} V_{1 B}\right) d t=\frac{1}{R_{1} C_{1}} \int v_{2} d t
$$

Las corrientes en el nodo de la entrada inversora del op-amp U1C son

$$
\frac{v_{1}}{R_{3}}-\frac{A_{1} v_{1} v_{2}}{R_{5}}+\frac{v_{3}}{R_{4}}+C_{3} \frac{d v_{3}}{d t}=0
$$

Despejando se obtiene

$$
\frac{d v_{3}}{d t}=\frac{1}{C_{3}}\left(-\frac{v_{1}}{R_{3}}-\frac{v_{3}}{R_{4}}+\frac{A_{1} v_{1} v_{2}}{R_{5}}\right)
$$

Por lo tanto, partiendo de las ecuaciones (20), (21) y (22), el circuito electrónico de la figura 4 es modelado por

$$
\left\{\begin{array}{l}
\frac{d v_{1}}{d t}=\frac{1}{R_{1} C_{1}}\left(v_{2}\right) \\
\frac{d v_{2}}{d t}=\frac{1}{R_{2} C_{2}}\left(v_{3}\right) \\
\frac{d v_{3}}{d t}=\frac{1}{R_{3} C_{3}}\left(-\frac{R_{3}}{R_{4}} v_{3}-v_{1}+\frac{A_{1} R_{3}}{R_{5}} v_{1} v_{2}\right)
\end{array}\right.
$$

El modelo (23) es equivalente al sistema caótico de Malasoma adimensional (19), bajo las condiciones y mapeos del tiempo, estados y parámetros dados por $\tau=\frac{1}{R_{1} C_{1}} t, \frac{v_{1}}{x_{1}}=\frac{v_{2}}{x_{2}}=\frac{v_{3}}{x_{3}}=M, M=\frac{R_{5}}{A_{1} R_{3}}, R_{1}=R_{2}=R_{3}, R_{6}=R_{7}$, $C_{1}=C_{2}=C_{3}, \alpha=\frac{R_{3}}{R_{4}}$. 


\section{Circuito MACM}

El sistema caótico MACM fue propuesto más recientemente ${ }^{20}$ como un sistema no lineal cuadrático, representado en su forma adimensional como

$$
\left\{\begin{array}{l}
\frac{d x_{1}}{d \tau}=-a x_{1}-b x_{2} x_{3} \\
\frac{d x_{2}}{d \tau}=-x_{1}+c x_{2} \\
\frac{d x_{3}}{d \tau}=d-x_{2}^{2}-x_{3}
\end{array}\right.
$$

donde $x_{1}, x_{2}$ y $x_{3}$ son las variables de estado, $\tau$ es una variable normalizada de tiempo, $a, b, c$ y $d$ son parámetros constantes. El sistema (24) tiene una realización electrónica por medio del circuito de la figura 5 , donde $R, R_{0}, R_{1}, R_{2}, \ldots, R_{11}$ son resistencias, $C_{1}, C_{2}$ y $C_{3}$ son capacitancias, $A_{1}$ y $A_{2}$ son las ganancias de los multiplicadores; $v_{1}, v_{2}$ y $v_{3}$ son los voltajes de salida de los op-amp U1B, U1D y U2B, respectivamente. $V_{C C}$ y $V_{S S}$ son los voltajes de alimentación de los amplificadores operacionales U1A, U1B, U1C, U1D, U2A y U2B.

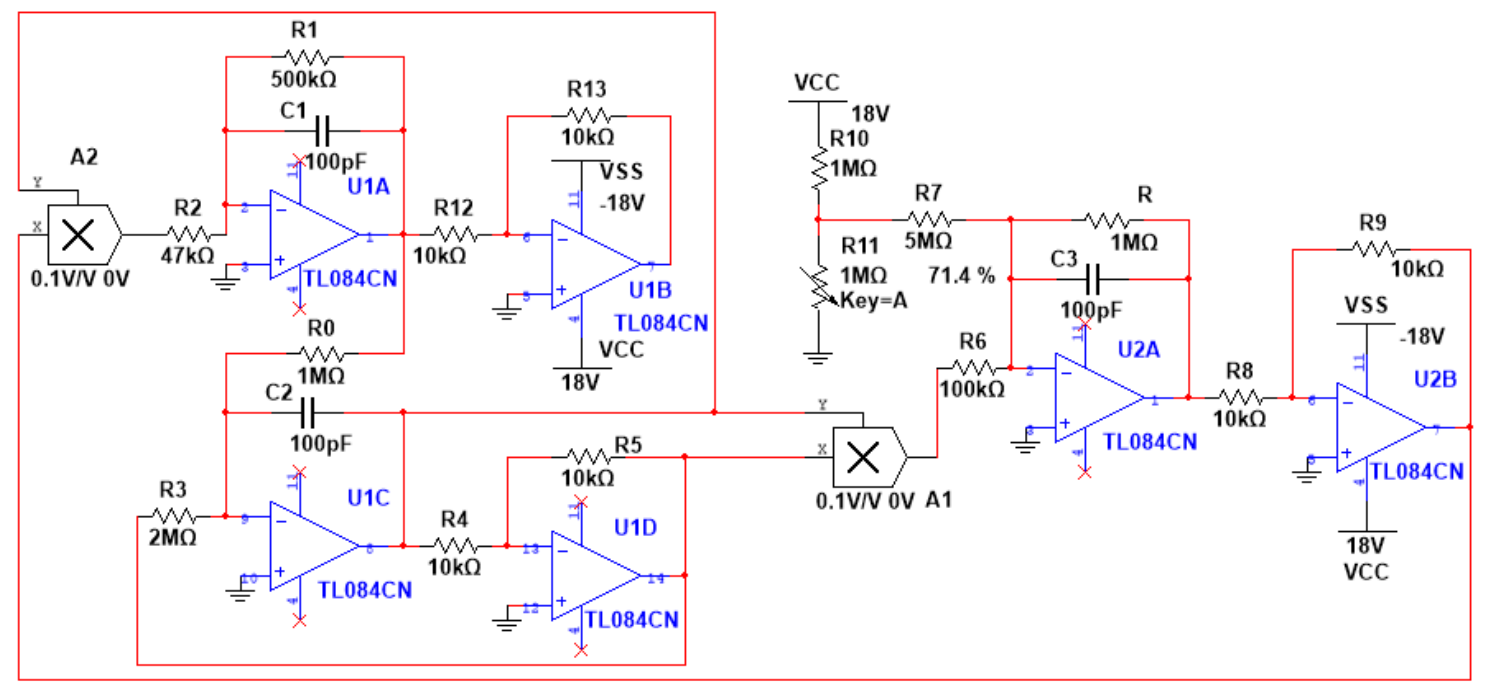

Fig. 5. Diagrama esquemático de realización electrónica para el sistema MACM.

Para demostrar la equivalencia del circuito de la figura 5 con el sistema (24) se realiza el siguiente análisis: Iniciando con el op-amp U1A, su voltaje de salida es

$$
V_{1 A}=-\frac{1}{C_{1}} \int\left(\frac{1}{R_{1}} V_{1 A}+\frac{A_{2}}{R_{2}} V_{1 C} v_{3}\right) d t
$$

Si $R_{12}=R_{13}$, el voltaje de salida del op-amp U1B es

$$
\begin{gathered}
v_{1}=-\frac{R_{13}}{R_{12}} V_{1 A}=-V_{1 A} \\
v_{1}=\frac{1}{C_{1}} \int\left(-\frac{1}{R_{1}} v_{1}+\frac{A_{2}}{R_{2}} V_{1 C} v_{3}\right) d t
\end{gathered}
$$

Para el op-amp U1C, su voltaje de salida es

$$
V_{1 C}=-\frac{1}{C_{2}} \int\left(\frac{1}{R_{3}} v_{2}+\frac{1}{R_{0}} V_{1 A}\right) d t=-\frac{1}{C_{2}} \int\left(\frac{1}{R_{3}} v_{2}-\frac{1}{R_{0}} v_{1}\right) d t
$$

Si $R_{4}=R_{5}$, el voltaje de salida del op-amp U1D es

$$
v_{2}=-\frac{R_{5}}{R_{4}} V_{1 C}=-V_{1 C}
$$




$$
v_{2}=\frac{1}{C_{2}} \int\left(\frac{1}{R_{3}} v_{2}-\frac{1}{R_{0}} v_{1}\right) d t
$$

Para el op-amp U2A, su voltaje de salida es

$$
V_{2 A}=-\frac{1}{C_{3}} \int\left(\frac{R_{11}}{R_{7}\left(R_{10}+R_{11}\right)} V_{C C}+\frac{A_{1}}{R_{6}} V_{1 C} v_{2}+\frac{1}{R} V_{2 A}\right) d t
$$

Si $R_{8}=R_{9}$, el voltaje de salida del op-amp U2B es

$$
\begin{gathered}
v_{3}=-\frac{R_{9}}{R_{8}} V_{2 A}=-V_{2 A} \\
v_{3}=\frac{1}{C_{3}} \int\left(\frac{R_{11}}{R_{7}\left(R_{10}+R_{11}\right)} V_{C C}-\frac{A_{1}}{R_{6}} v_{2}^{2}-\frac{1}{R} v_{3}\right) d t
\end{gathered}
$$

Por lo tanto, partiendo de las ecuaciones (25), (26) y (27), si $R_{0}=R$, el circuito electrónico de la figura 5 es modelado por

$$
\left\{\begin{array}{l}
\frac{d v_{1}}{d t}=\frac{1}{R C_{1}}\left(-\frac{R}{R_{1}} v_{1}-\frac{A_{2} R}{R_{2}} v_{2} v_{3}\right) \\
\frac{d v_{2}}{d t}=\frac{1}{R C_{2}}\left(-v_{1}+\frac{R}{R_{3}} v_{2}\right) \\
\frac{d v_{3}}{d t}=\frac{1}{R C_{3}}\left(\frac{R R_{11}}{R_{7}\left(R_{10}+R_{11}\right)} V_{C C}-\frac{A_{1} R}{R_{6}} v_{2}^{2}-v_{3}\right)
\end{array}\right.
$$

El modelo (28) es equivalente al sistema caótico de MACM adimensional (24), bajo las condiciones y mapeos del tiempo, estados y parámetros dados por $\tau=\frac{1}{R C_{1}} t, \frac{v_{1}}{x_{1}}=\frac{v_{2}}{x_{2}}=\frac{v_{3}}{x_{3}}=M, M=\frac{R_{6}}{A_{1} R}, R_{0}=R, R_{4}=R_{5}, R_{8}=R_{9}$, $R_{12}=R_{13}, C_{1}=C_{2}=C_{3}, a=\frac{R}{R_{1}}, b=\frac{A_{2} R_{6}}{A_{1} R_{2}}, c=\frac{R}{R_{3}}$ y $d=\frac{A_{1} R^{2} R_{11}}{R_{6} R_{7}\left(R_{10}+R_{11}\right)} V_{C C}$.

\section{DISEÑO DE EXPERIMENTOS}

Con el fin de verificar que el comportamiento de las realizaciones electrónicas sea congruente con el comportamiento típico de los sistemas caóticos de Lorenz, Lü, Chen, Rössler, Liu-Chen, Malasoma y MACM, los circuitos analógicos de las figuras 1 a 5 son implementados en el software de circuitos Multisim y su respuesta se compara con la de los modelos adimensionales en simulaciones numéricas en el software Scilab.

Para la instrumentación electrónica del circuito analógico de Lorenz de la figura 1 se utilizaron op-amp LM324 y los circuitos integrados AD633JN como multiplicadores de voltaje cuya ganancia fue de $A_{1}=A_{2}=1 \mathrm{~V} / \mathrm{V}$, se consideraron los parámetros $R_{1}=R_{2}=R_{3}=R_{4}=R_{7}=R_{8}=R_{10}=R_{12}=R_{13}=100 \mathrm{k} \Omega, R_{5}=10 \mathrm{k} \Omega, R_{6}=$ $3.57 \mathrm{k} \Omega, \quad R_{9}=R_{11}=1.5 \mathrm{k} \Omega, R_{14}=37.5 \mathrm{k} \Omega, \mathrm{C}_{1}=\mathrm{C}_{2}=\mathrm{C}_{3}=10 \mathrm{nF}, S_{0}=1$ y $S_{1}=1 ;$ que corresponden a los valores $\sigma=10, \rho=28$ y $b=8 / 3$ del modelo adimensional (2), con $M=0.015$ y $\tau=1,000 t$.

Para la instrumentación electrónica del circuito analógico de Lü de la figura 1 se utilizaron op-amp LM324 y los circuitos integrados AD633JN como multiplicadores de voltaje cuya ganancia fue de $A_{1}=A_{2}=1 \mathrm{~V} / \mathrm{V}$, se consideraron los parámetros $R_{1}=R_{2}=R_{3}=R_{4}=R_{8}=R_{10}=R_{12}=R_{13}=100 \mathrm{k} \Omega, R_{5}=2.77 \mathrm{k} \Omega, R_{6}$ no importa, $R_{7}=5 \mathrm{k} \Omega, R_{9}=R_{11}=1.5 \mathrm{k} \Omega, R_{14}=33.3 \mathrm{k} \Omega, \mathrm{C}_{1}=\mathrm{C}_{2}=\mathrm{C}_{3}=10 \mathrm{nF}, S_{0}=0$ y $S_{1}=0$; que corresponden a los valores $a=36, b=3$ y $c=20$ del modelo adimensional (3), con $M=0.015$ y $\tau=1,000 t$.

Para la instrumentación electrónica del circuito analógico de Chen de la figura 1 se utilizaron op-amp LM324 y los circuitos integrados AD633JN como multiplicadores de voltaje cuya ganancia fue de $A_{1}=A_{2}=1 \mathrm{~V} / \mathrm{V}$, se consideraron los parámetros $R_{1}=R_{2}=R_{3}=R_{4}=R_{8}=R_{10}=R_{12}=R_{13}=100 \mathrm{k} \Omega, R_{5}=2.857 \mathrm{k} \Omega, R_{6}=$ 
$14.286 \mathrm{k} \Omega, \quad R_{7}=3.57 \mathrm{k} \Omega, R_{9}=R_{11}=1.5 \mathrm{k} \Omega, R_{14}=33.3 \mathrm{k} \Omega, \mathrm{C}_{1}=\mathrm{C}_{2}=\mathrm{C}_{3}=10 \mathrm{nF}, S_{0}=1$ y $S_{1}=0 ;$ que corresponden a los valores $a=35, b=3$ y $c=28$ del modelo adimensional (4), con $M=0.015$ y $\tau=1,000 t$.

Para la instrumentación electrónica del circuito analógico de Rössler de la figura 2 se utilizaron op-amp TL082 y el circuito integrado AD633JN como multiplicador de voltaje cuya ganancia fue de $A_{1}=0.1 \mathrm{~V} / \mathrm{V}$. Se consideraron los parámetros $R_{1}=R_{3}=R_{4}=R_{5}=R_{6}=R_{10}=R_{11}=R_{12}=100 \mathrm{k} \Omega, R_{2}=500 \mathrm{k} \Omega, R_{7}=7.5 \mathrm{M} \Omega, R_{8}=$ $100 \Omega, R_{9}=10 \mathrm{k} \Omega, R_{13}=1.754 \mathrm{k} \Omega, \mathrm{C}_{1}=\mathrm{C}_{2}=\mathrm{C}_{3}=1 \mathrm{nF}, V_{C C}=15 \mathrm{~V}, V_{S S}=-15 \mathrm{~V}$, que corresponden a los valores $a=0.2, b=0.2$ y $c=5.7$ del modelo adimensional (9), con $M=0.1$ y $\tau=10,000 t$.

Para la instrumentación electrónica del circuito analógico de Liu-Chen de la figura 3 se utilizaron op-amp TL082 y TL084, AD633JN como multiplicadores de voltaje cuya ganancia fue de $A_{1}=A_{2}=A_{3}=10 \mathrm{~V} / \mathrm{V}$, y se consideraron los parámetros $R_{1}=R_{2}=R_{9}=R_{10}=R_{11}=R_{12}=10 \mathrm{k} \Omega, R_{3}=80 \mathrm{k} \Omega, R_{4}=R_{6}=R_{8}=$ $400 \mathrm{k} \Omega, R_{5}=40 \mathrm{k} \Omega, R_{7}=117.64 \mathrm{k} \Omega, C_{1}=C_{2}=C_{3}=1 \mathrm{nF}, V_{S S}=-15 \mathrm{~V}, V_{D D}=15 \mathrm{~V}$; que corresponden a los valores $a=5, b=10$ y $c=3.4$ del modelo adimensional (14), con $M=0.1$ y $\tau=2,500 t$.

Para la instrumentación electrónica del circuito analógico de Malasoma de la figura 4, se usaron op-amp TL084, el circuito integrado AD633JN como multiplicador de voltaje cuya ganancia fue de $A_{1}=0.1 \mathrm{~V} / \mathrm{V}$ y se consideraron los parámetros $R_{1}=R_{2}=R_{3}=R_{6}=R_{7}=10 \mathrm{k} \Omega, R_{4}=4.9 \mathrm{k} \Omega, R_{5}=560 \Omega, C_{1}=C_{2}=C_{3}=$ $100 \mathrm{nF}$ y voltajes de alimentación de $V_{C C}=12 \mathrm{~V}$ y $V_{S S}=-12 \mathrm{~V}$, que corresponden al valor $\alpha=\frac{R_{1}}{R_{4}}=2.04 \mathrm{del}$ modelo adimensional (19), con $M=0.56$ y $\tau=10,000 t$.

Para la instrumentación electrónica del circuito analógico MACM de la figura 5, se usaron op-amp TL084, circuitos integrados AD633JN como multiplicadores de voltaje cuyas ganancias fueron de $A_{1}=A_{2}=0.1 \mathrm{~V} / \mathrm{V} \mathrm{y}$ se consideraron los parámetros $R_{0}=R=1 \mathrm{M} \Omega, R_{1}=500 \mathrm{k} \Omega, R_{2}=47 \mathrm{k} \Omega, R_{3}=2 \mathrm{M} \Omega, R_{4}=R_{5}=R_{8}=R_{9}=$ $R_{12}=R_{13}=10 \mathrm{k} \Omega, R_{6}=100 \mathrm{k} \Omega, R_{7}=5 \mathrm{M} \Omega, R_{10}=1 \mathrm{M} \Omega, R_{11}=714 \mathrm{k} \Omega, \mathrm{C}_{1}=\mathrm{C}_{2}=\mathrm{C}_{3}=100 \mathrm{pF}$, voltajes de alimentación de $V_{C C}=18 \mathrm{~V}$ y $V_{S S}=-18 \mathrm{~V}$, que corresponden a los valores $a=2, b=2.13, c=0.5$ y $d=$ 1.5 del modelo adimensional (24), con $M=1$ y $\tau=10,000 t$.

\section{RESULTADOS DE SIMULACIONES Y DE IMPLEMENTACIÓN}

A continuación, se muestran y comparan los resultados obtenidos de las implementaciones de los circuitos y simulaciones de los modelos adimensionales. Los datos numéricos obtenidos se muestran gráficamente en forma de proyecciones de los atractores caóticos en los planos de fase.

\section{Sistema de Lorenz}

En la figura 6, se muestran los voltajes del circuito analógico de la figura 1 en proyecciones planares de su atractor caótico con Multisim. En la figura 7, se muestra el atractor del modelo (8), obtenido para ese circuito.
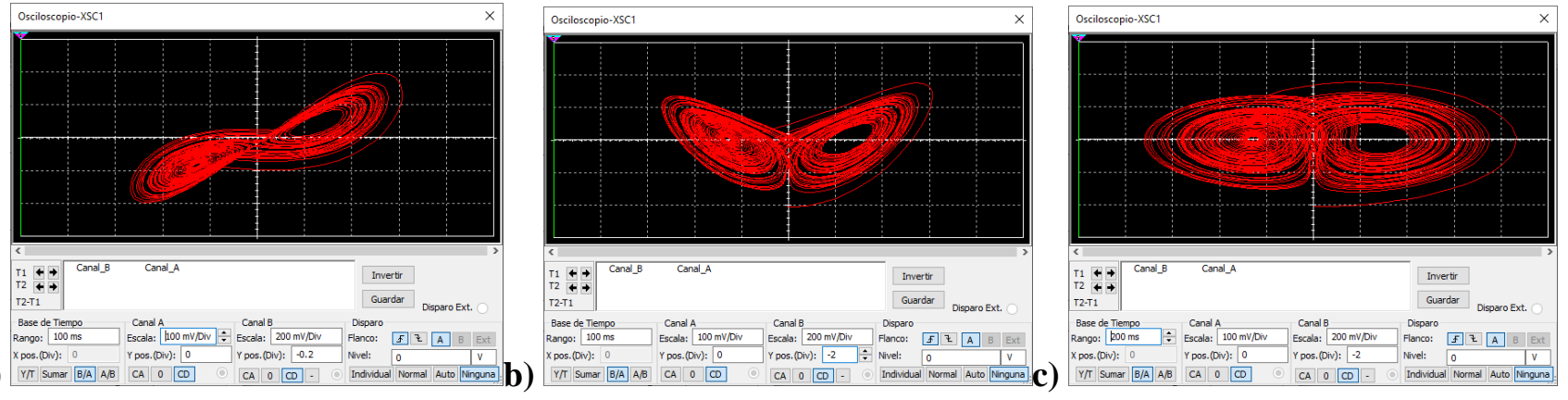

Fig. 6. Atractor de Lorenz del circuito de la figura 1, en planos $v_{1}-v_{2}(a), v_{1}-v_{3}$ (b) y $v_{2}-v_{3}$ (c). 

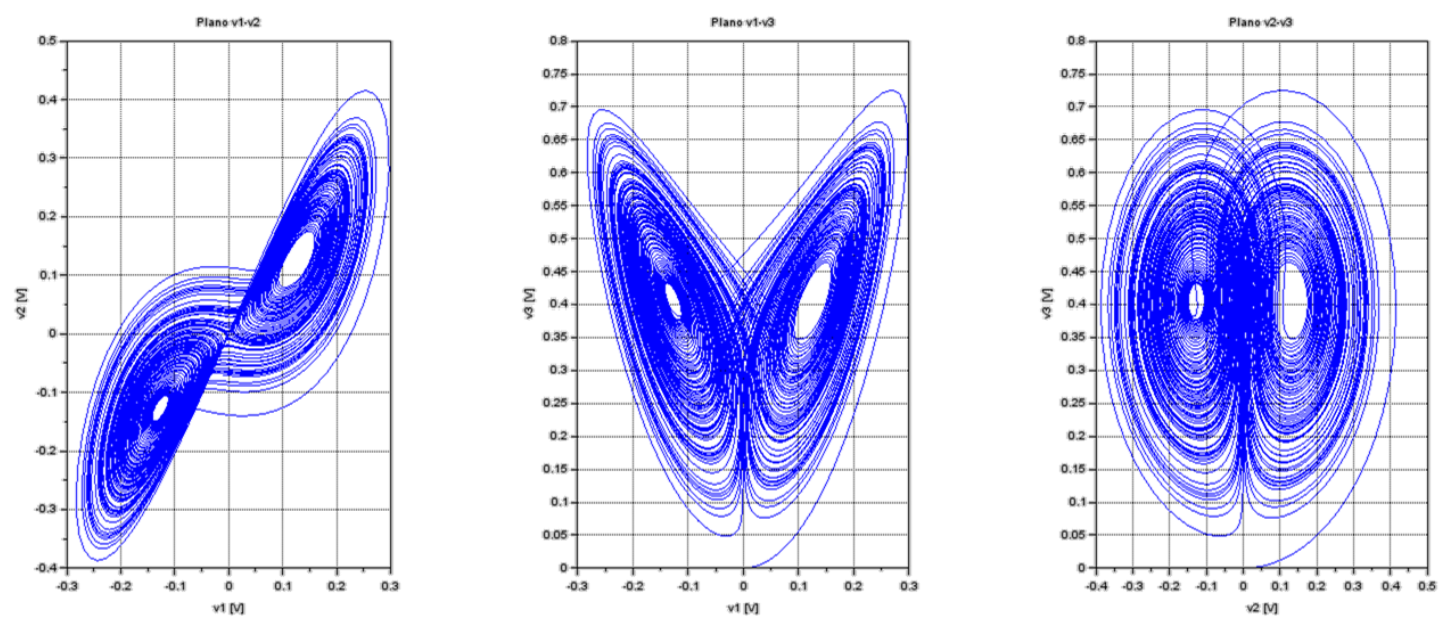

Fig. 7. Atractor de Lorenz del modelo (8) del circuito, en planos $v_{1}-v_{2}, v_{1}-v_{3}$ y $v_{2}-v_{3}$.

En la figura 8, se muestra el atractor del modelo (8) mapeado a la escala equivalente del sistema adimensional (2). En la figura 9, se muestra el atractor del sistema adimensional (2) mediante el software Scilab.
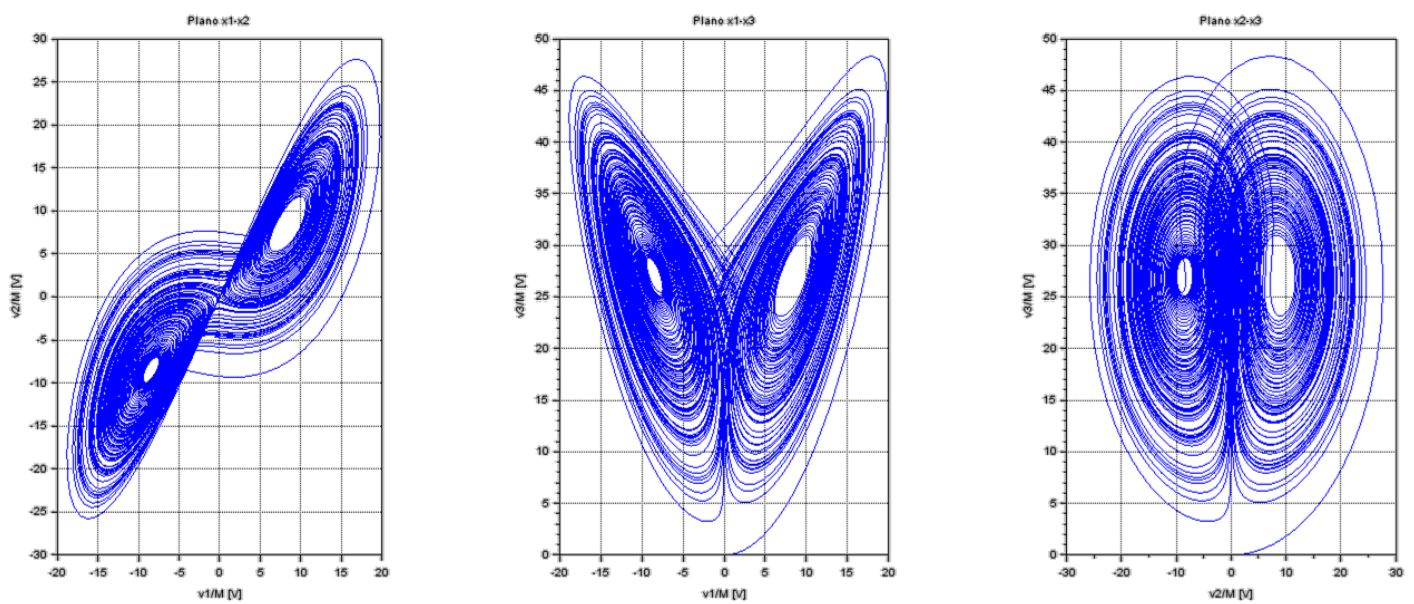

Fig. 8. Atractor de Lorenz del modelo (8) mapeado a escala $x_{1}, x_{2}, x_{3}$, en planos $x_{1}-x_{2}, x_{1}-x_{3}$ y $x_{2}-x_{3}$.
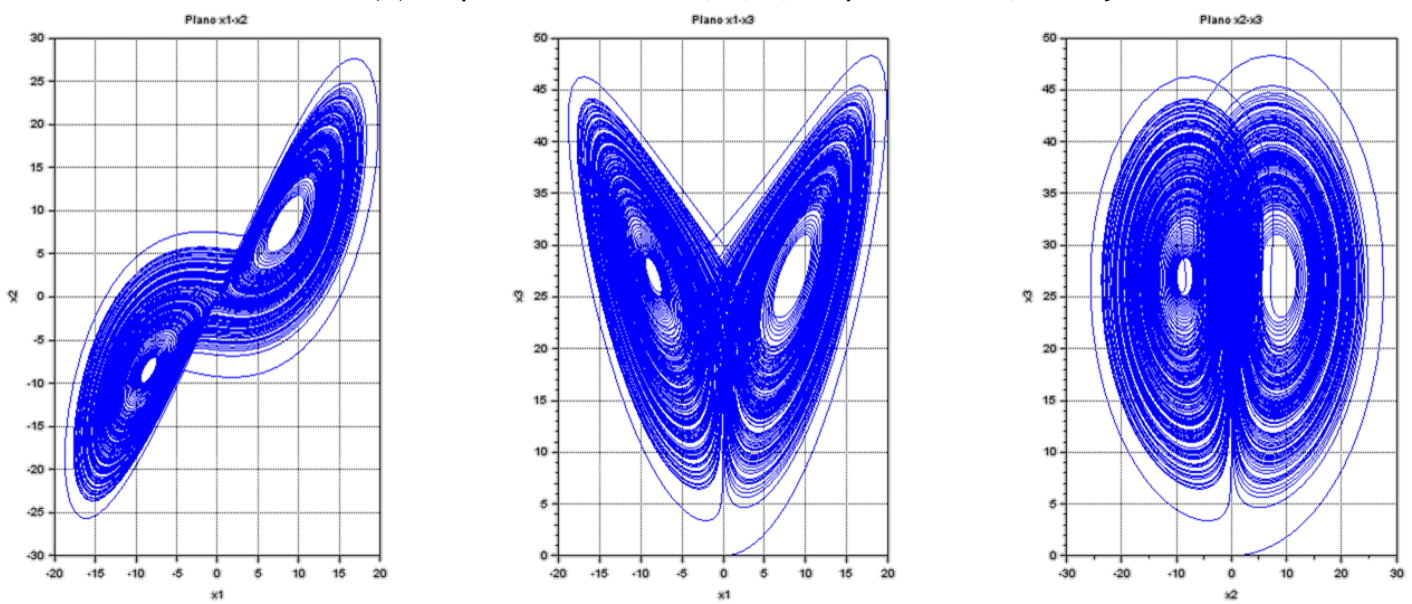

Fig. 9. Atractor de Lorenz del modelo adimensional (2), en planos $x_{1}-x_{2}, x_{1}-x_{3}$ y $x_{2}-x_{3}$.

\section{Sistema de Lü}

En la figura 10, se muestran los voltajes del circuito analógico de la figura 1 en proyecciones planares de su atractor caótico con Multisim. En la figura 11, se muestra el atractor del modelo (8), obtenido para ese circuito. En la figura 
12, se muestra el atractor del modelo (8) mapeado a la escala equivalente del sistema adimensional (3). En la figura 13, se muestra el atractor del sistema adimensional (3) mediante el software Scilab.
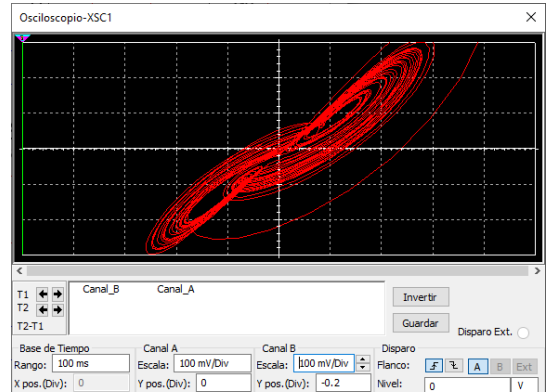
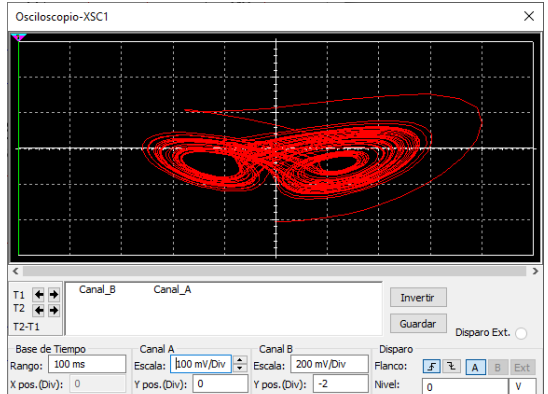
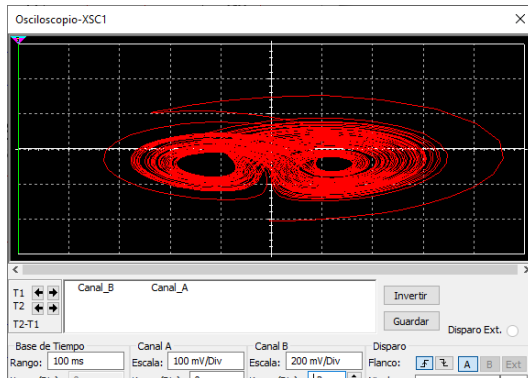

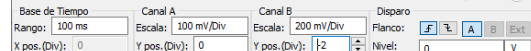

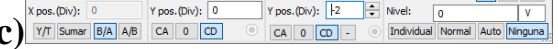

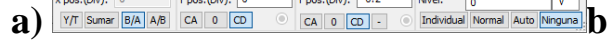

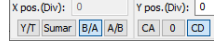

0 - -0 Individud Normal Auto Ninguna:

(c).
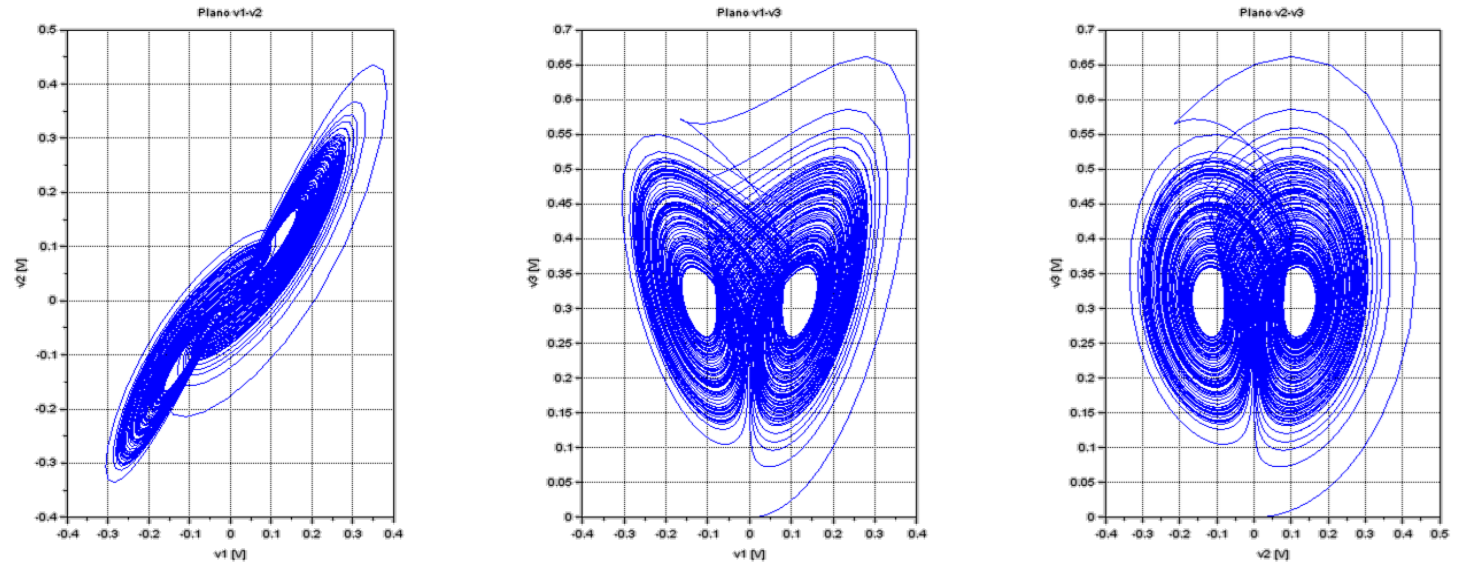

Fig. 11. Atractor de Lü del modelo (8) del circuito, en planos $v_{1}-v_{2}, v_{1}-v_{3}$ y $v_{2}-v_{3}$.
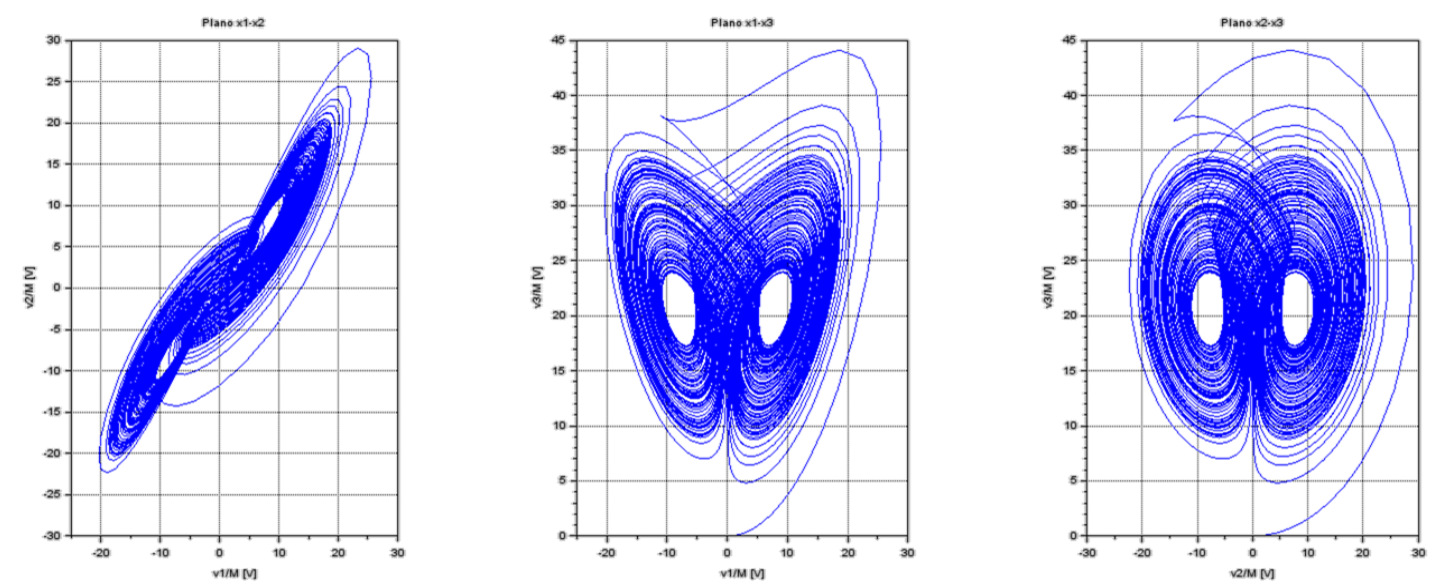

Fig. 12. Atractor de Lü del modelo (8) mapeado a escala $x_{1}, x_{2}, x_{3}$, en planos $x_{1}-x_{2}, x_{1}-x_{3}$ y $x_{2}-x_{3}$. 

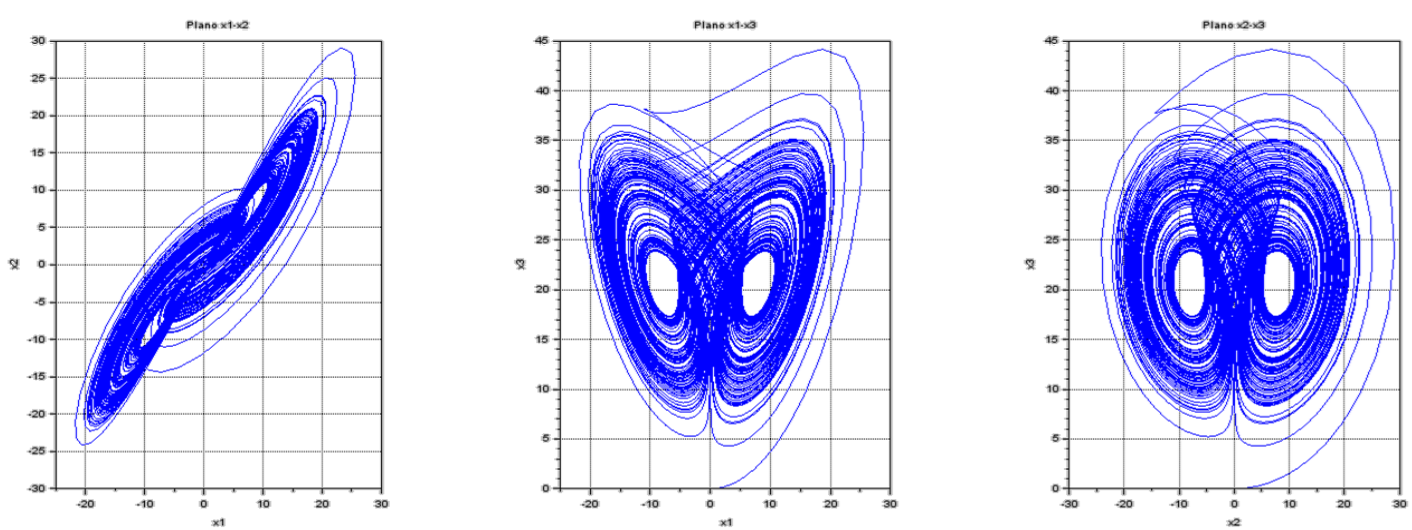

Fig. 13. Atractor de Lü del modelo adimensional (3), en planos $x_{1}-x_{2}, x_{1}-x_{3}$ y $x_{2}-x_{3}$.

\section{Sistema de Chen}

En la figura 14, se muestran los voltajes del circuito analógico de la figura 1 en proyecciones planares de su atractor caótico con Multisim. En la figura 15, se muestra el atractor del modelo (8), obtenido para ese circuito. En la figura 16, se muestra el atractor del modelo (8) mapeado a la escala equivalente del sistema adimensional (4). En la figura 17, se muestra el atractor del sistema adimensional (4) mediante el software Scilab.
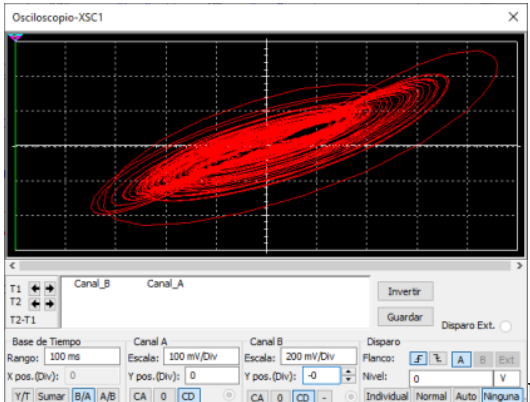
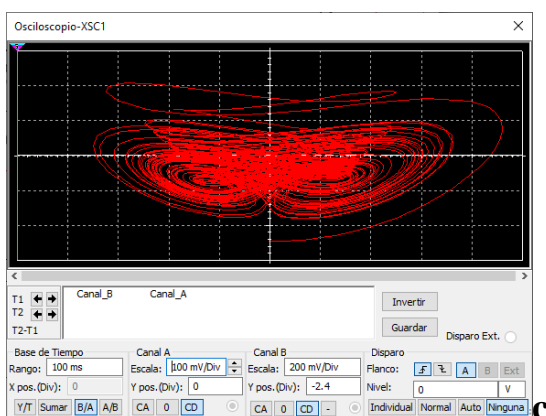
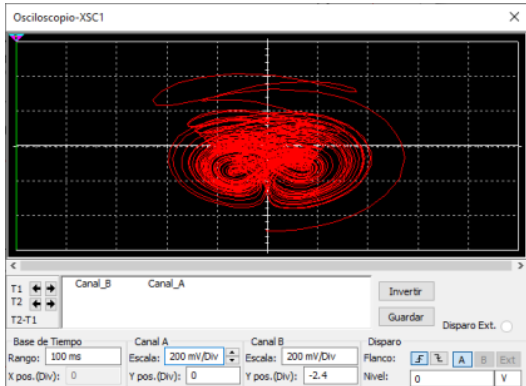

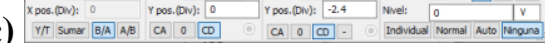

Fig. 14. Atractor de Chen del circuito de la figura 1, en planos $v_{1}-v_{2}$ (a), $v_{1}-v_{3}$ (b) y $v_{2}-v_{3}$ (c).
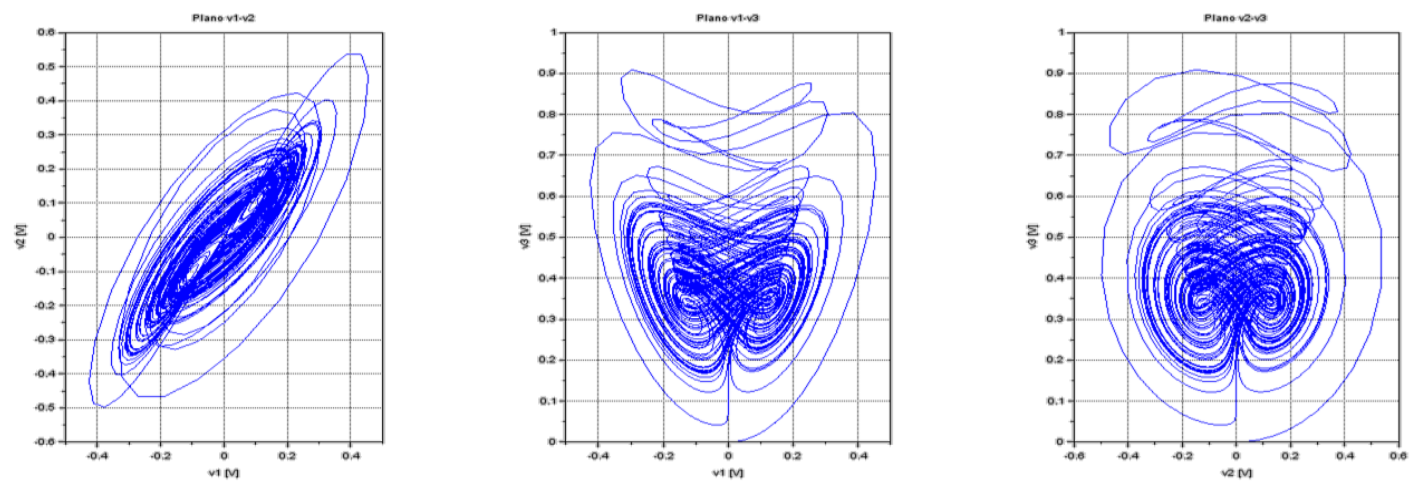

Fig. 15. Atractor de Chen del modelo (8) del circuito, en planos $v_{1}-v_{2}, v_{1}-v_{3}$ y $v_{2}-v_{3}$. 

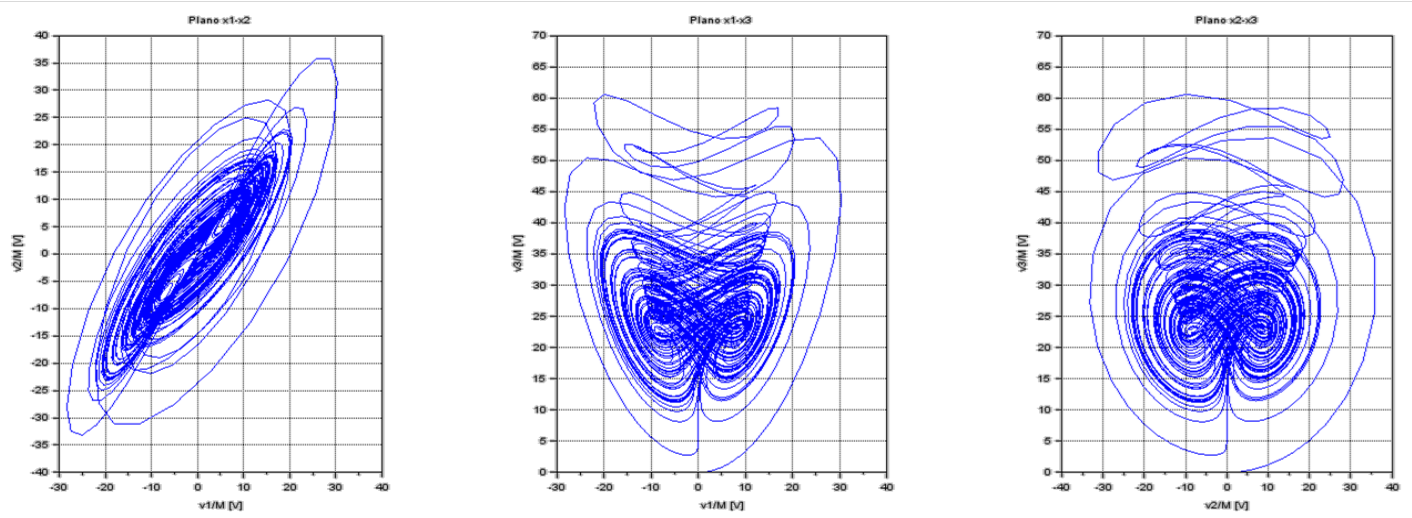

Fig. 16. Atractor de Chen del modelo (8) mapeado a escala $x_{1}, x_{2}, x_{3}$, en planos $x_{1}-x_{2}, x_{1}-x_{3}$ y $x_{2}-x_{3}$.
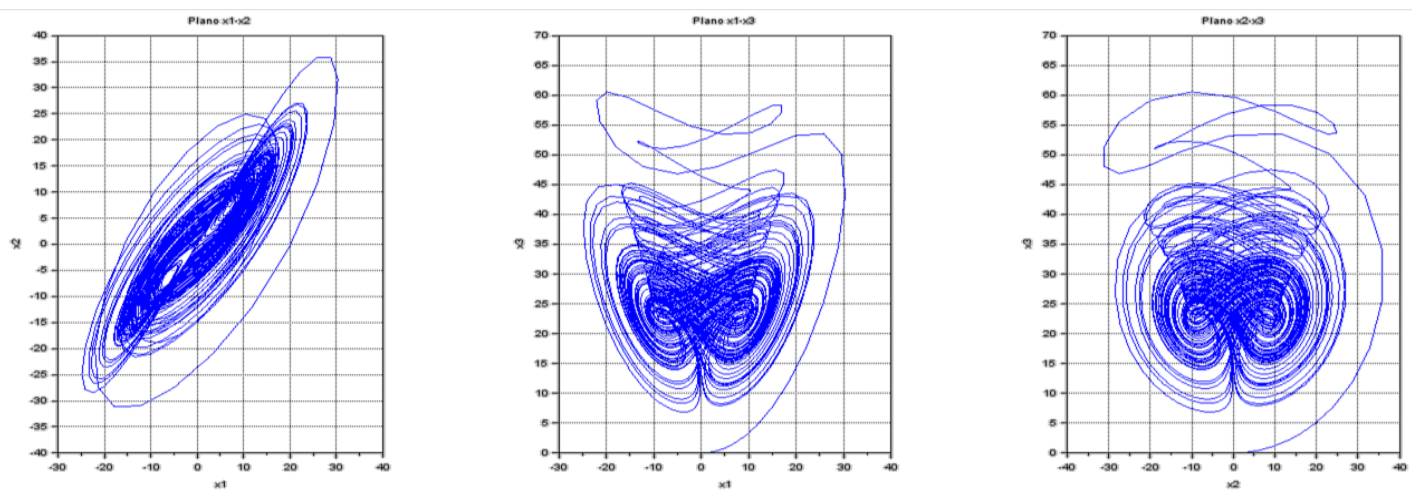

Fig. 17. Atractor de Chen del modelo adimensional (4), en planos $x_{1}-x_{2}, x_{1}-x_{3}$ y $x_{2}-x_{3}$.

\section{Sistema de Rössler}

En la figura 18, se muestran los voltajes del circuito analógico de la figura 2 en proyecciones planares de su atractor caótico con Multisim. En la figura 19, se muestra el atractor del modelo (13), obtenido para ese circuito. En la figura 20, se muestra el atractor del modelo (13) mapeado a la escala equivalente del sistema adimensional (9). En la figura 21, se muestra el atractor del sistema adimensional (9) mediante el software Scilab.

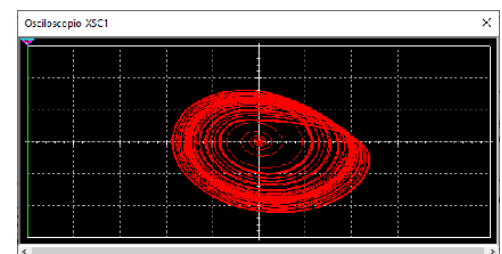

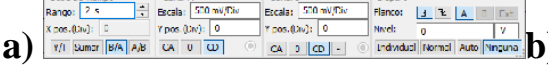

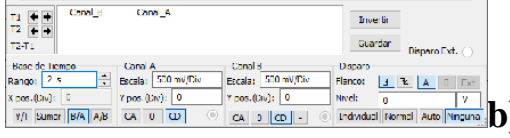

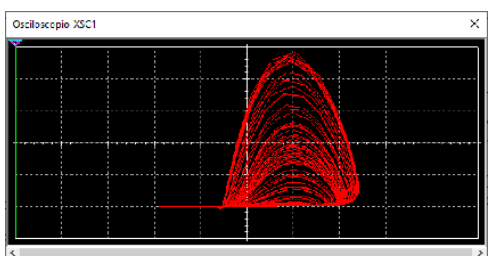

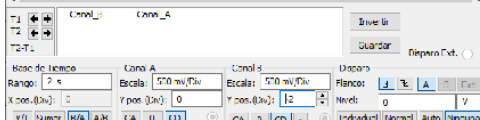

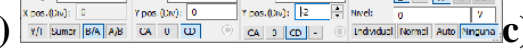
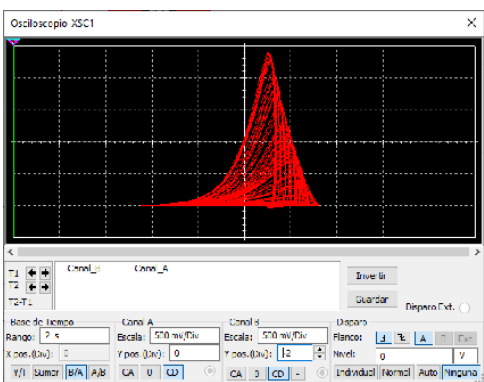

Fig. 18. Atractor de Rössler del circuito de la figura 2, en planos $v_{1}-v_{2}(a), v_{1}-v_{3}$ (b) y $v_{2}-v_{3}$ (c). 

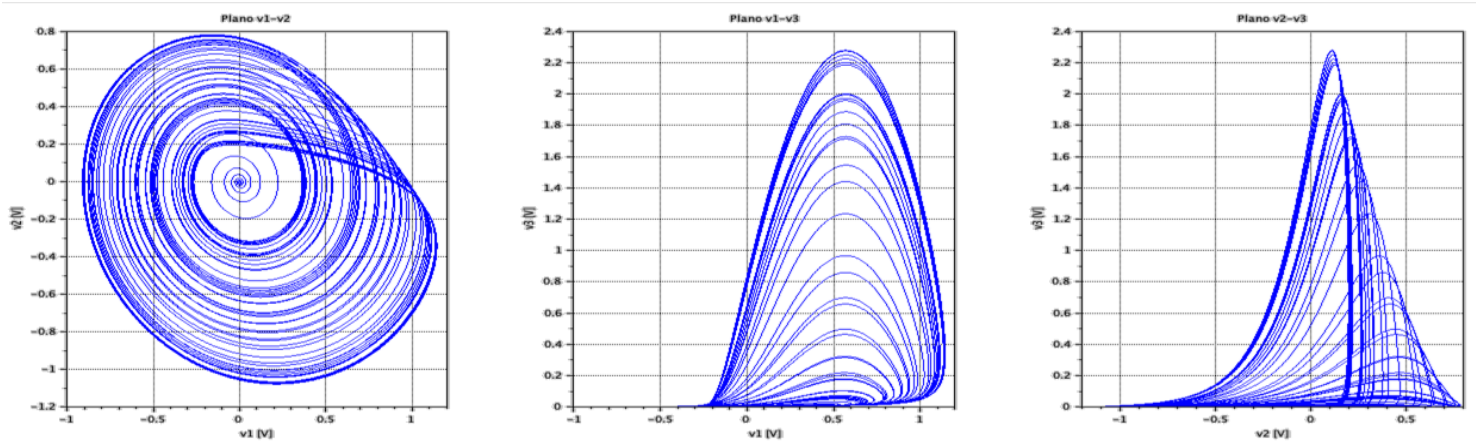

Fig. 19. Atractor de Rössler del modelo (13) del circuito, en planos $v_{1}-v_{2}, v_{1}-v_{3}$ y $v_{2}-v_{3}$.
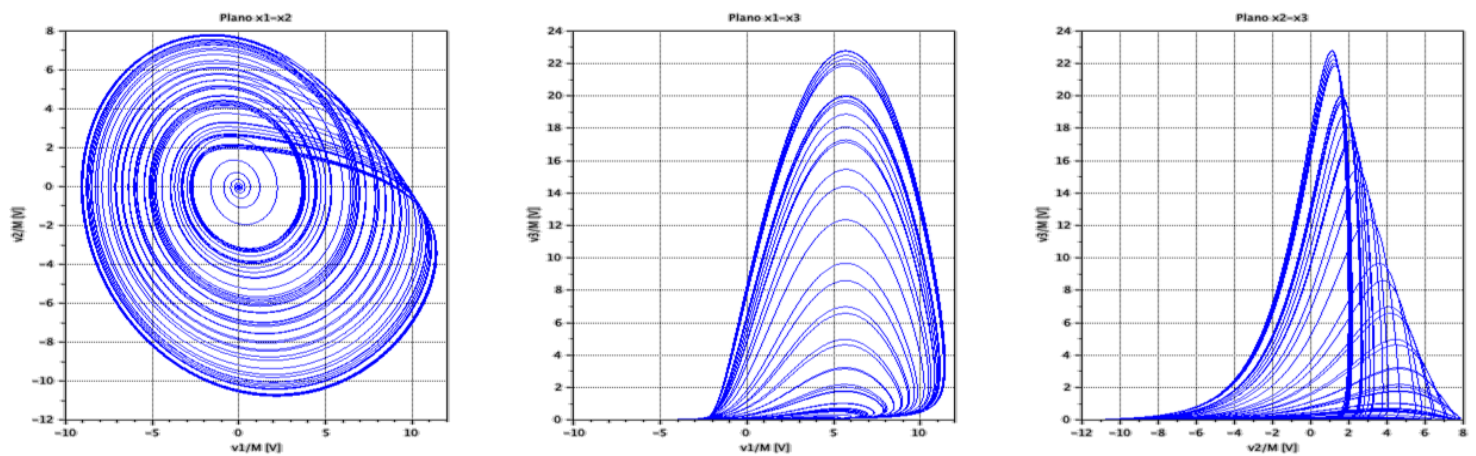

Fig. 20. Atractor de Rössler del modelo (13) mapeado a escala $x_{1}, x_{2}, x_{3}$, en planos $x_{1}-x_{2}, x_{1}-x_{3}$ y $x_{2}-x_{3}$.
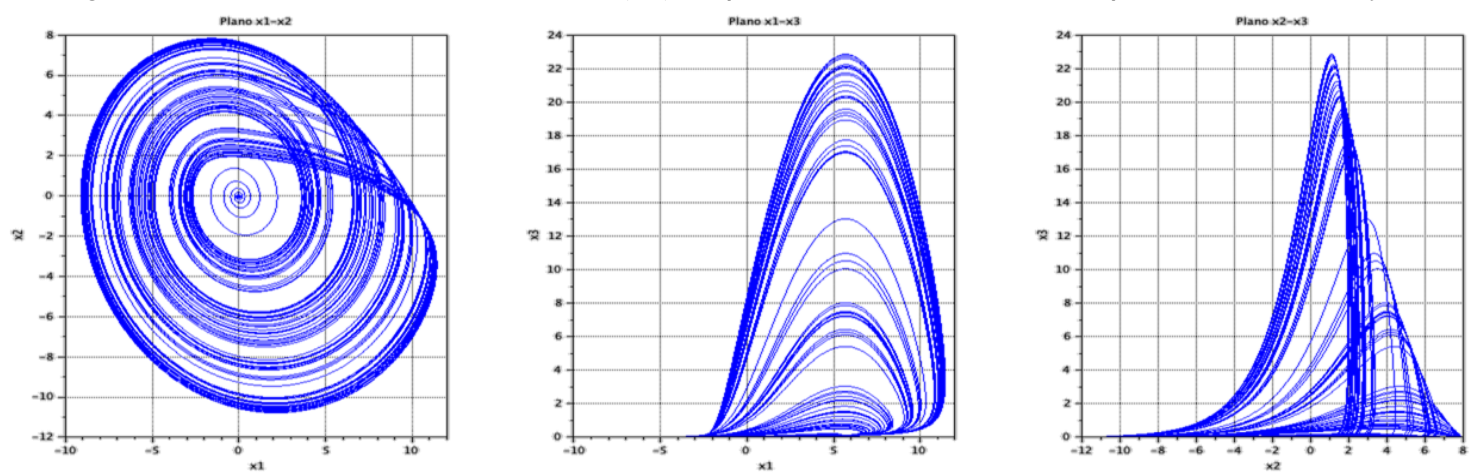

Fig. 21. Atractor de Rössler del modelo adimensional (9), en planos $x_{1}-x_{2}, x_{1}-x_{3}$ y $x_{2}-x_{3}$.

\section{Sistema Liu-Chen}

En la figura 22, se muestran los voltajes del circuito analógico de la figura 3 en proyecciones planares de su atractor caótico con Multisim. En la figura 23, se muestra el atractor del modelo (18), obtenido para ese circuito. En la figura 24, se muestra el atractor del modelo (18) mapeado a la escala equivalente del sistema adimensional (14). En la figura 25, se muestra el atractor del sistema adimensional (14) mediante el software Scilab.
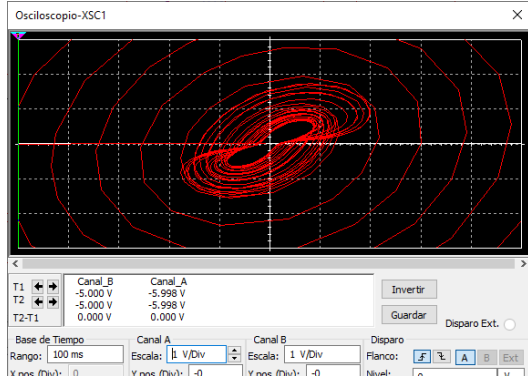

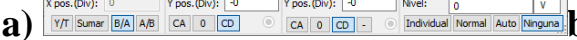
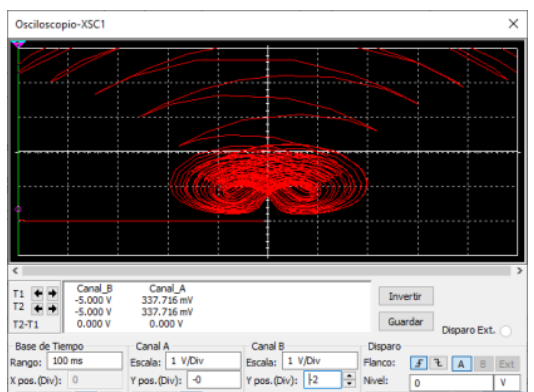

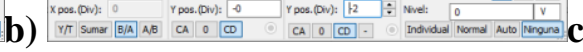

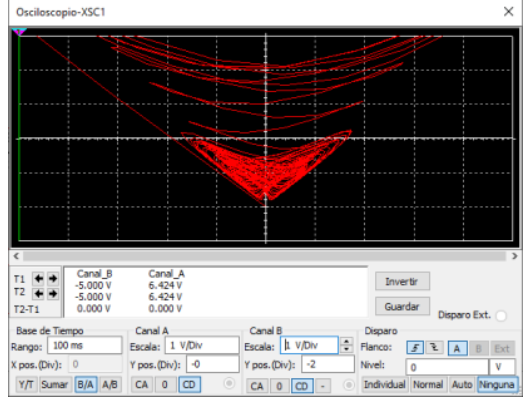

Fig. 22. Atractor de Liu-Chen del circuito de la figura 3, en planos $v_{1}-v_{2}(a), v_{1}-v_{3}$ (b) y $v_{2}-v_{3}$ (c). 

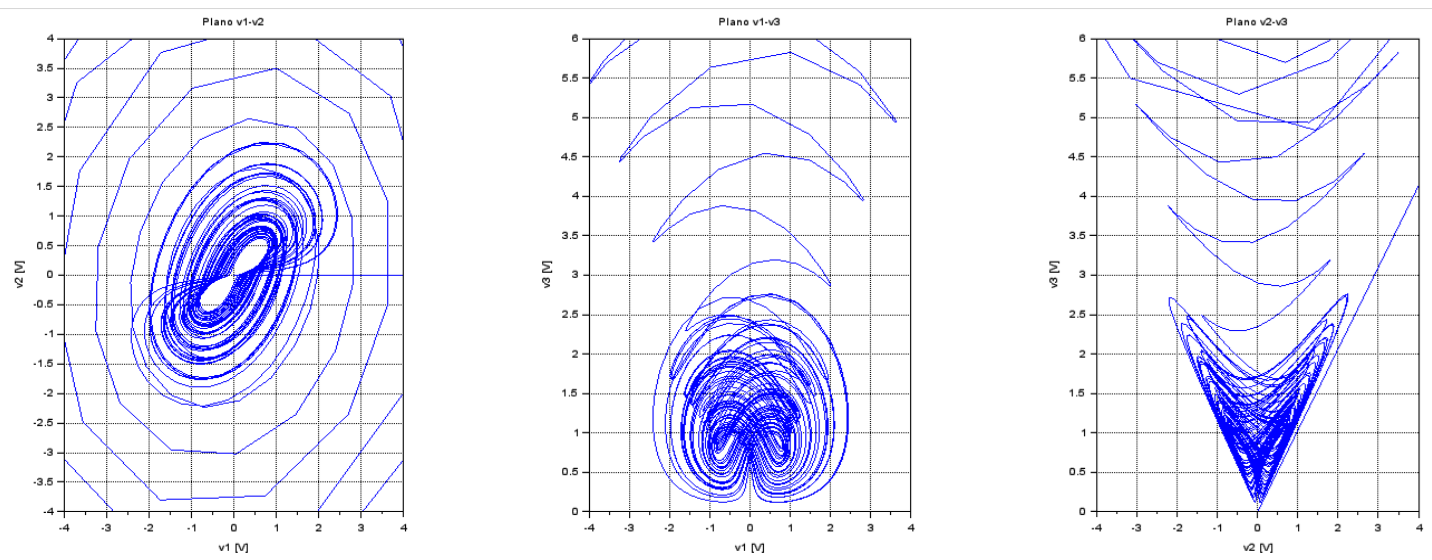

Fig. 23. Atractor de Liu-Chen del modelo (18) del circuito, en planos $v_{1}-v_{2}, v_{1}-v_{3}$ y $v_{2}-v_{3}$.
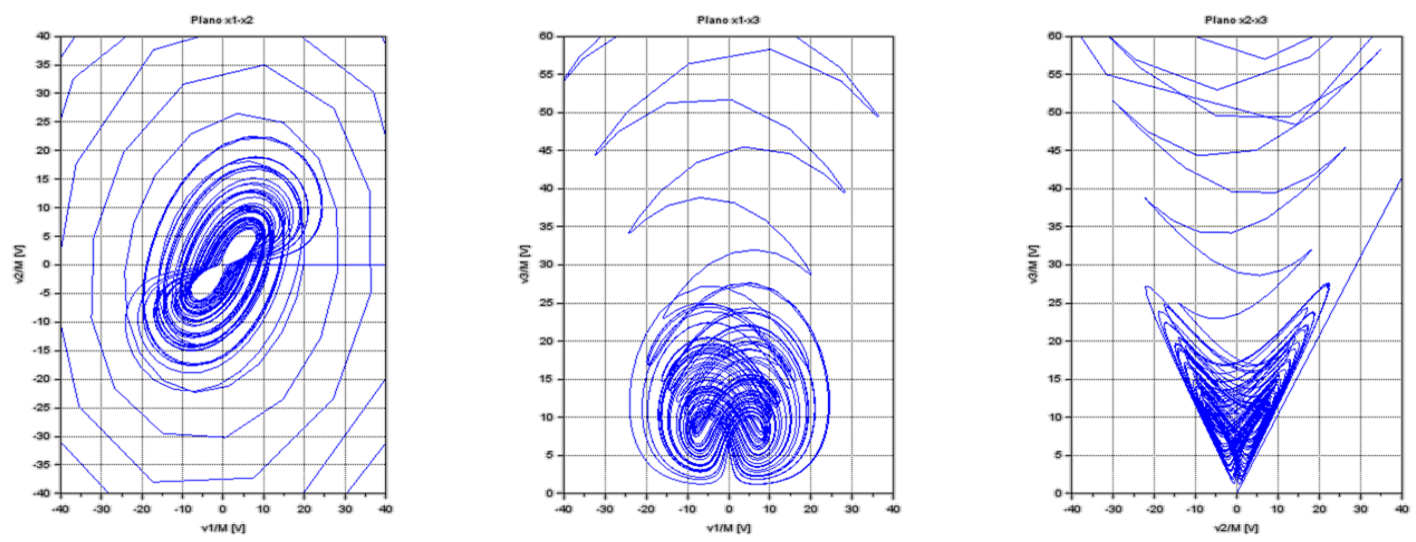

Fig. 24. Atractor de Liu-Chen del modelo (18) mapeado a escala $x_{1}, x_{2}, x_{3}$, en planos $x_{1}-x_{2}, x_{1}-x_{3}$ y $x_{2}-x_{3}$.
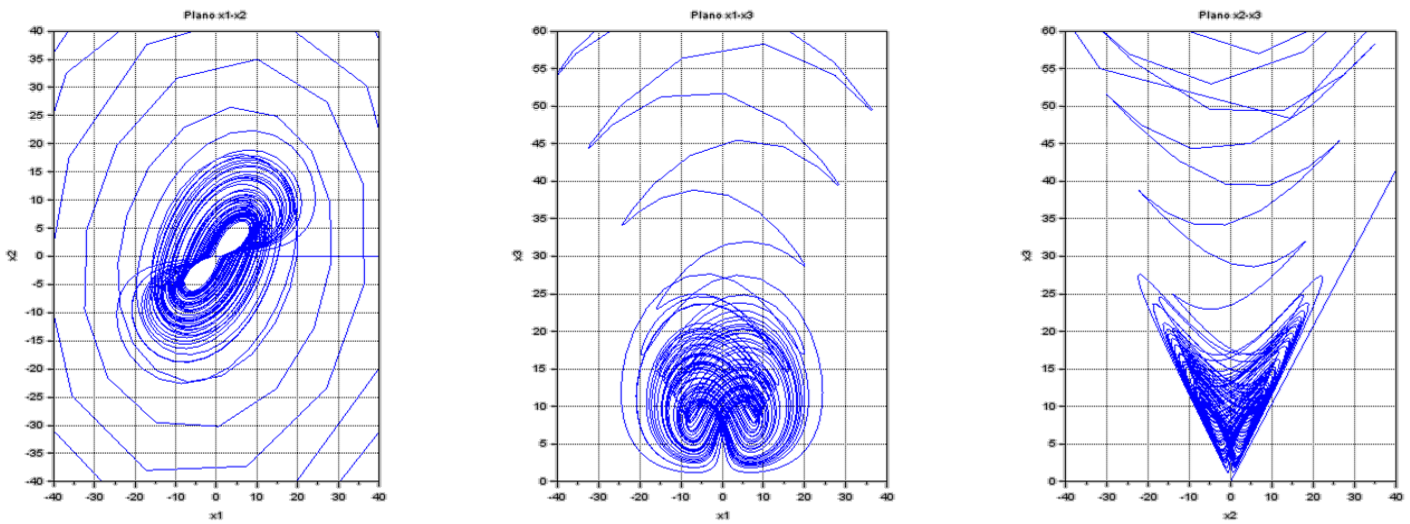

Fig. 25. Atractor de Liu-Chen del modelo adimensional (14), en planos $x_{1}-x_{2}, x_{1}-x_{3}$ y $x_{2}-x_{3}$.

\section{Sistema Malasoma}

En la figura 26, se muestran los voltajes del circuito analógico de la figura 4 en proyecciones planares de su atractor caótico con Multisim. En la figura 27, se muestra el atractor del modelo (23), obtenido para ese circuito. En la figura 28, se muestra el atractor del modelo (23) mapeado a la escala equivalente del sistema adimensional (19). En la figura 29, se muestra el atractor del sistema adimensional (19) mediante el software Scilab. 

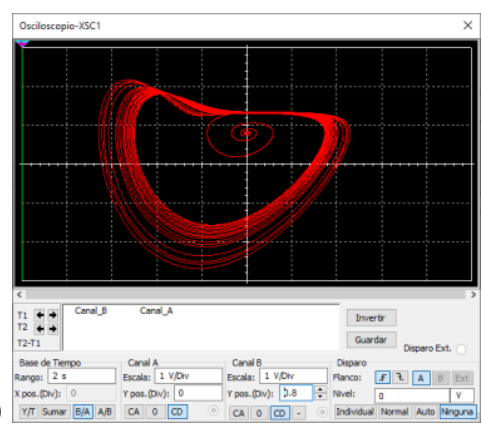
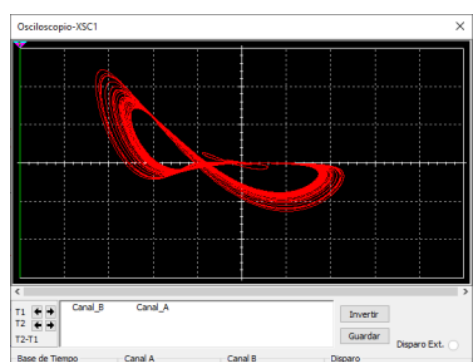

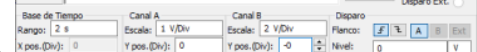
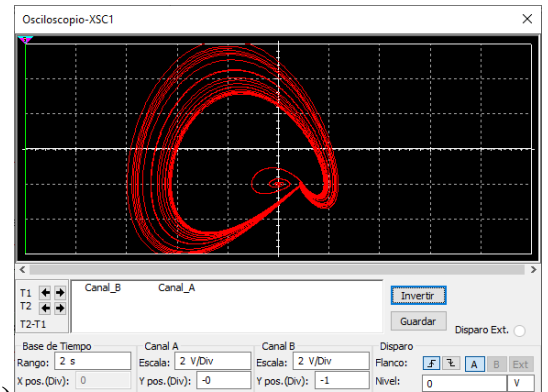

Fig. 26. Atractor de Malasoma del circuito de la figura 4, en planos $v_{1}-v_{2}$ (a), $v_{1}-v_{3}$ (b) y $v_{2}-v_{3}$ (c).
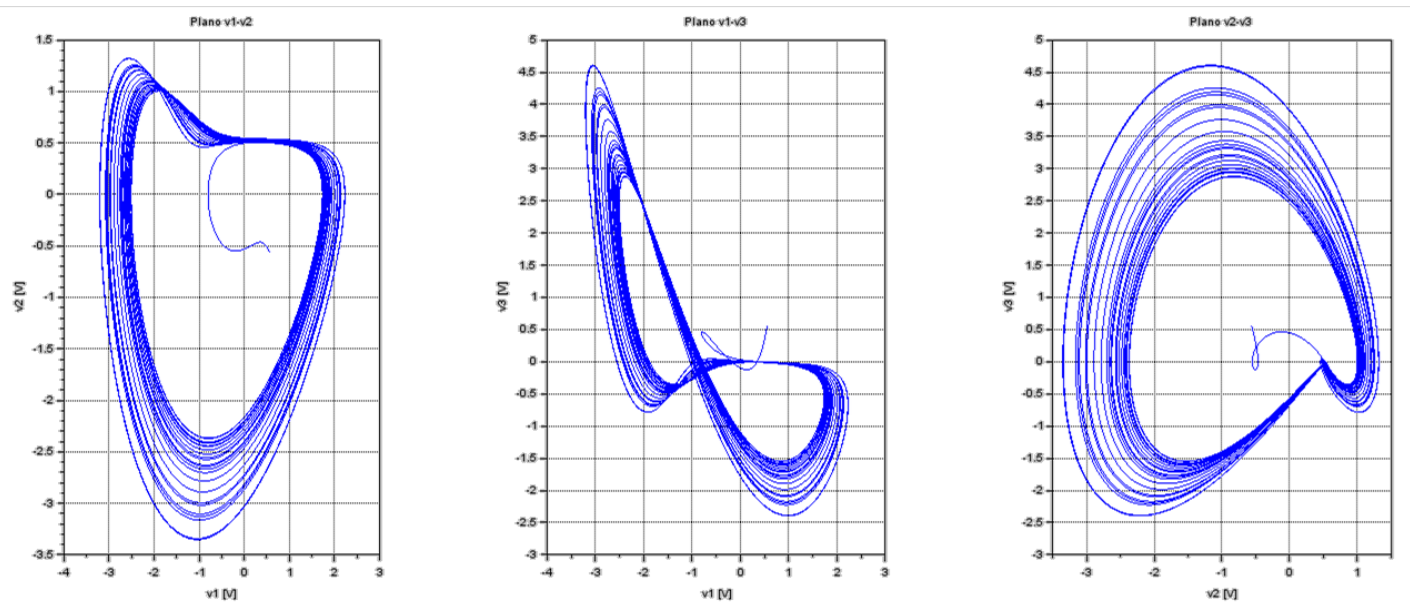

Fig. 27. Atractor de Malasoma del modelo (23) del circuito, en planos $v_{1}-v_{2}, v_{1}-v_{3}$ y $v_{2}-v_{3}$.
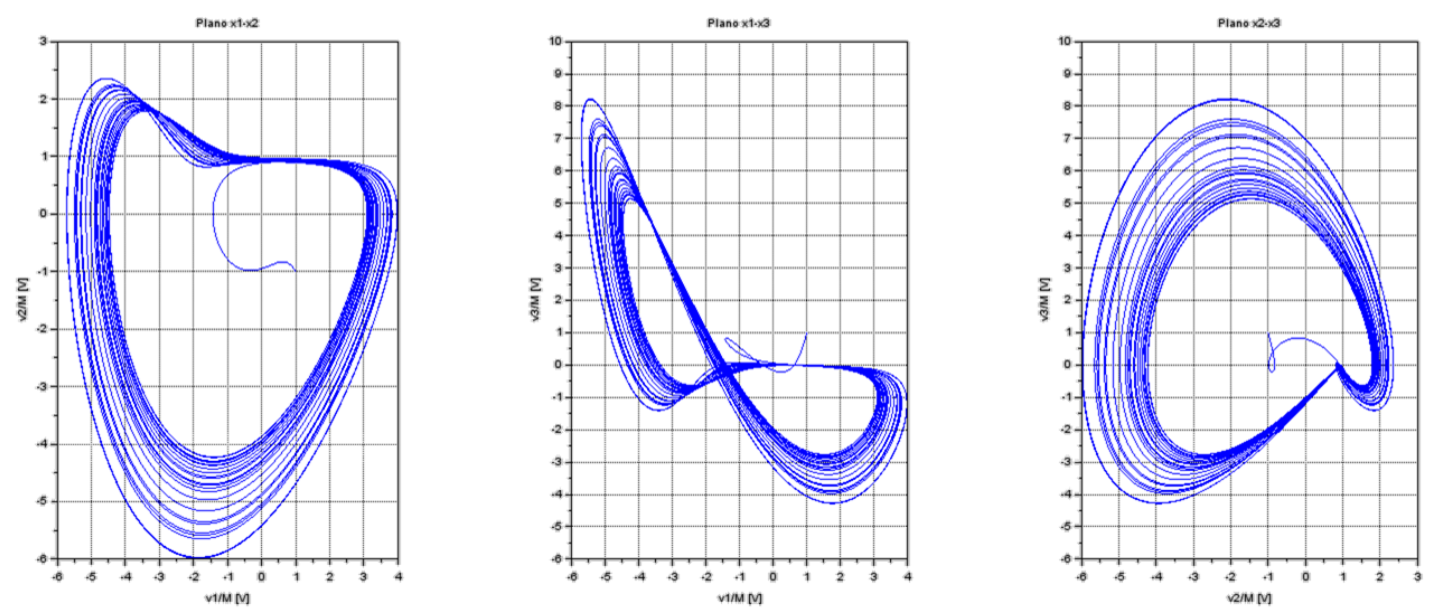

Fig. 28. Atractor de Malasoma del modelo (23) mapeado a escala $x_{1}, x_{2}, x_{3}$, en planos $x_{1}-x_{2}, x_{1}-x_{3}$ y $x_{2}-x_{3}$. 

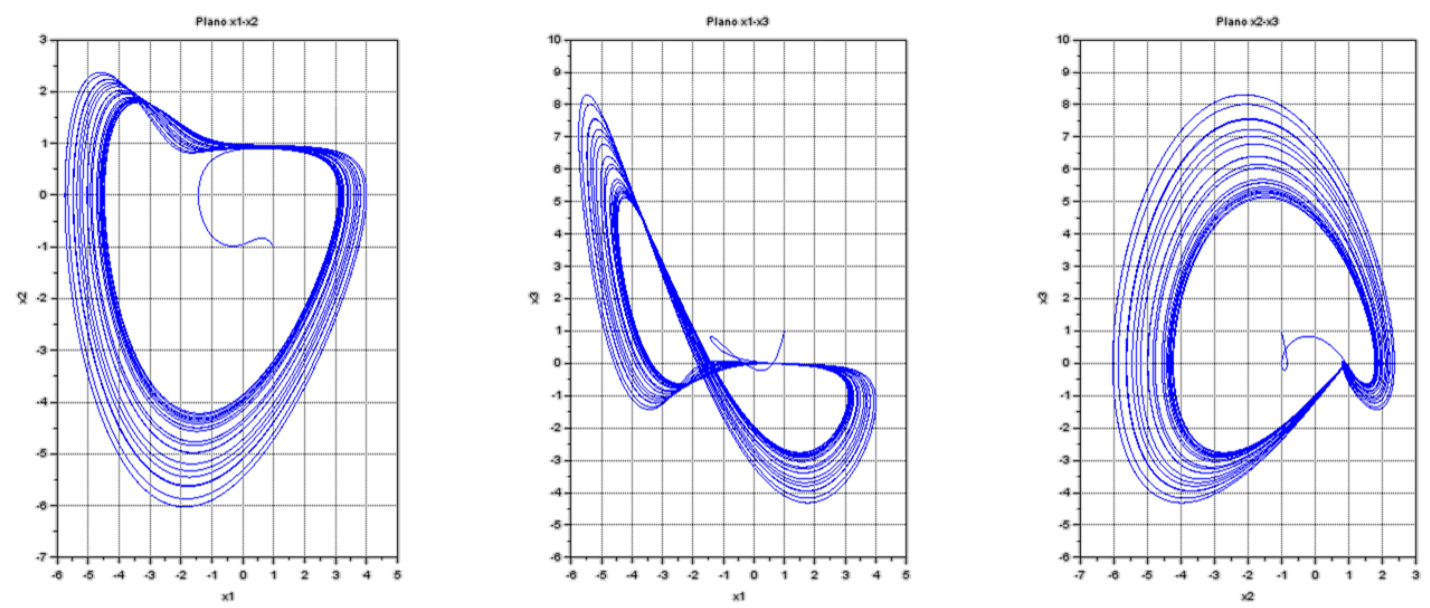

Fig. 29. Atractor de Malasoma del modelo adimensional (19), en planos $x_{1}-x_{2}, x_{1}-x_{3}$ y $x_{2}-x_{3}$.

\section{Sistema MACM}

En la figura 30, se muestran los voltajes del circuito analógico de la figura 5 en proyecciones planares de su atractor caótico con Multisim. En la figura 31, se muestra el atractor del modelo (28), obtenido para ese circuito. En la figura 32, se muestra el atractor del modelo (28) mapeado a la escala equivalente del sistema adimensional (24). En la figura 33, se muestra el atractor del sistema adimensional (24) mediante el software Scilab.
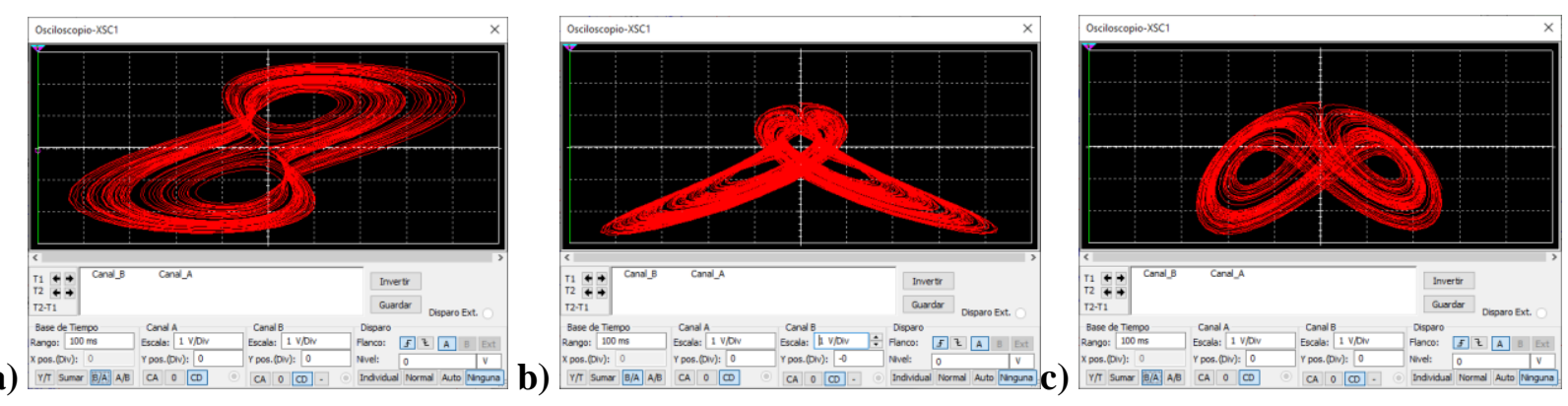

Fig. 30. Atractor de MACM del circuito de la figura 5, en planos $v_{1}-v_{2}$ (a), $v_{1}-v_{3}$ (b) y $v_{2}-v_{3}$ (c).
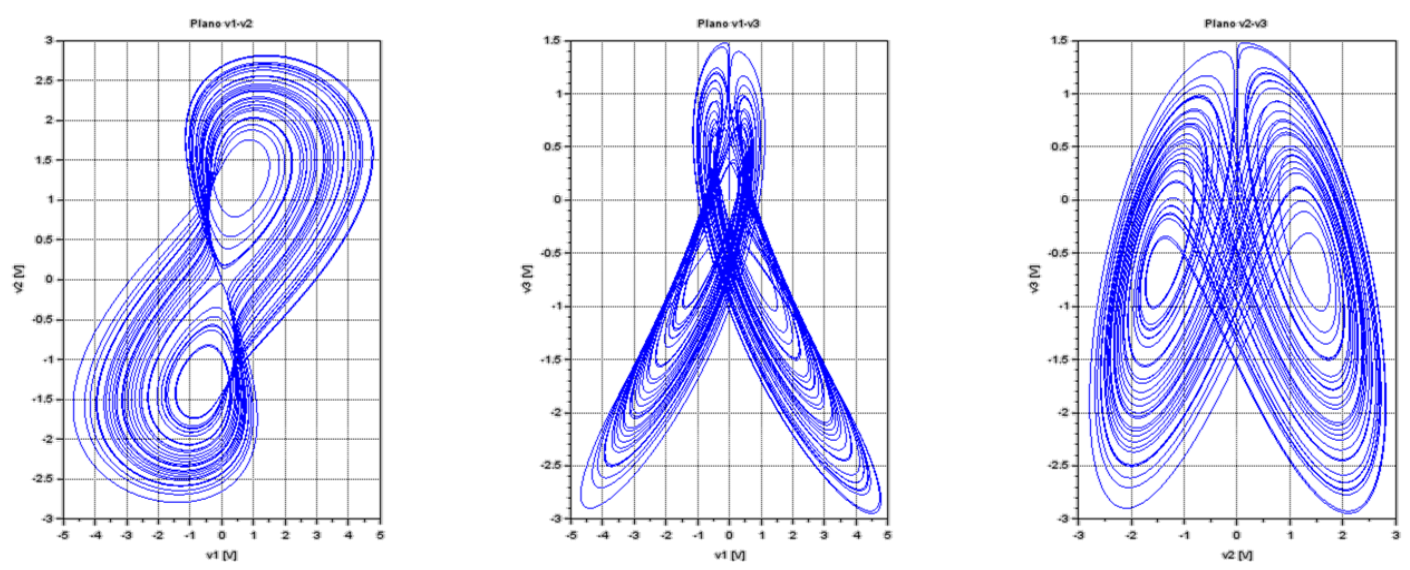

Fig. 31. Atractor de MACM del modelo (28) del circuito, en planos $v_{1}-v_{2}, v_{1}-v_{3}$ y $v_{2}-v_{3}$. 

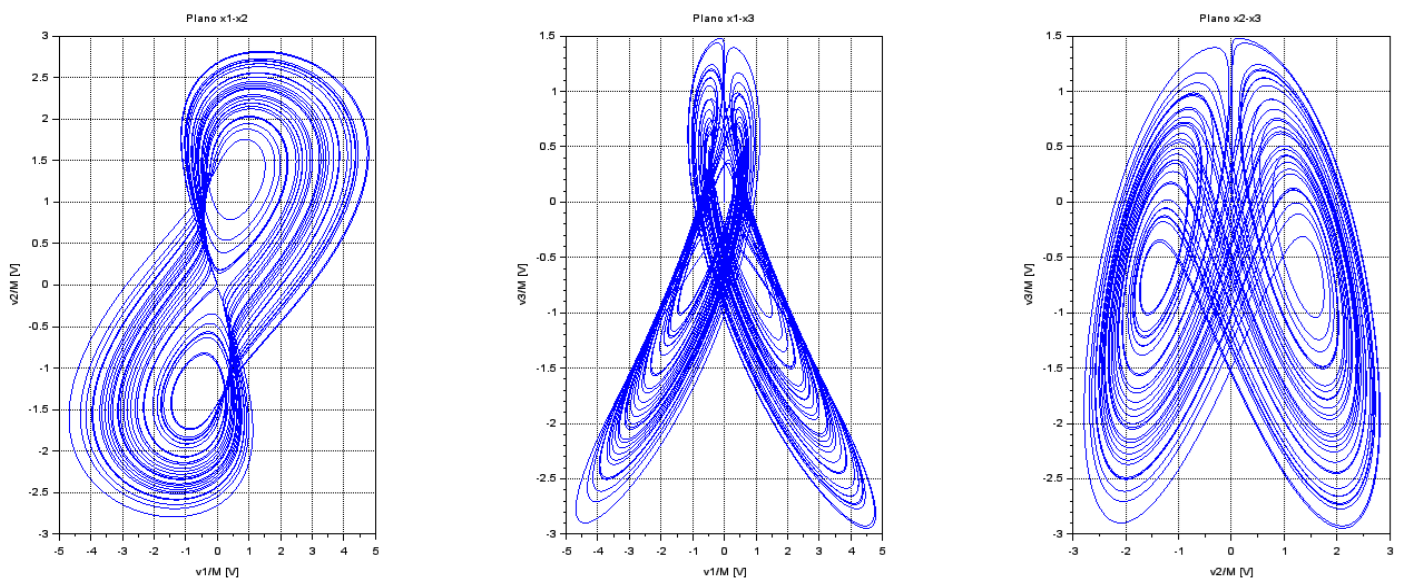

Fig. 32. Atractor de MACM del modelo (28) mapeado a escala $x_{1}, x_{2}, x_{3}$, en planos $x_{1}-x_{2}, x_{1}-x_{3}$ y $x_{2}-x_{3}$.
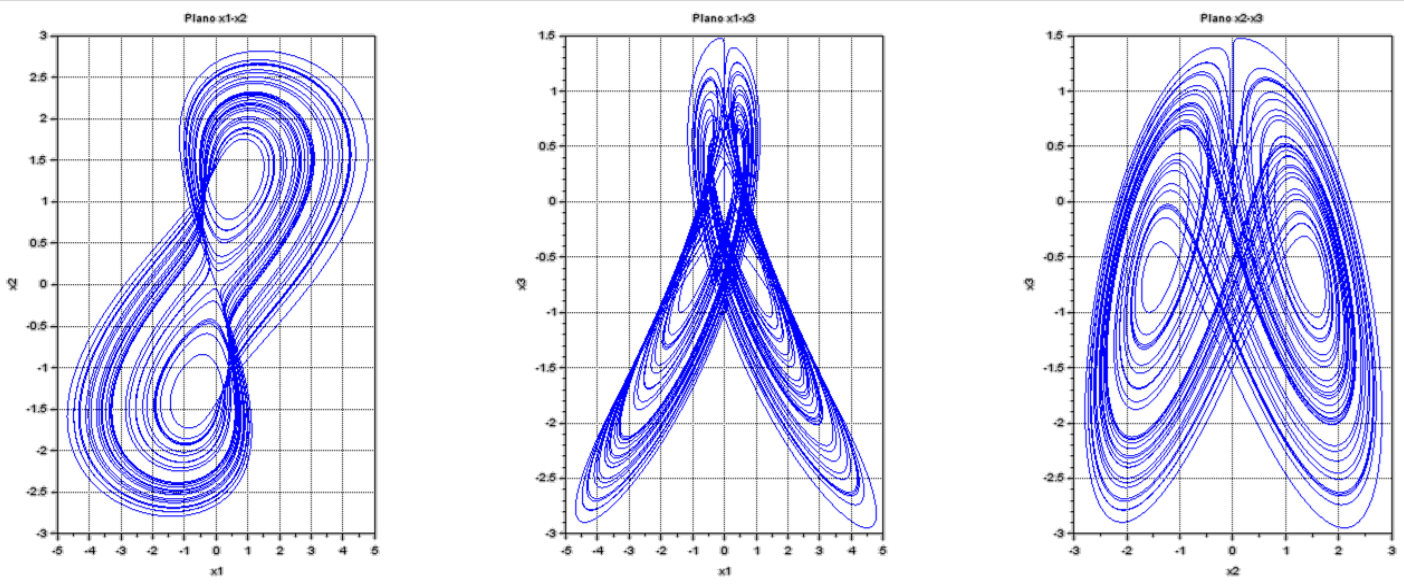

Fig. 33. Atractor de MACM del modelo adimensional (24), en planos $x_{1}-x_{2}, x_{1}-x_{3}$ y $x_{2}-x_{3}$.

\section{DISCUSIÓN DE RESULTADOS}

Como puede verse en las figuras $6,10,14,18,22,26$ y 30, los voltajes mostrados en osciloscopio del software Multisim reproducen los atractores caóticos respectivos, en rangos de operación admisibles. Con las figuras 7, 11, 15, 19, 23, 27 y 31, se valida la respuesta de los modelos obtenidos para los circuitos electrónicos respectivos (véase la forma y amplitud de cada atractor). En las figuras 8, 12, 16, 20, 24, 28 y 32, se puede apreciar que los atractores escalados de los modelos matemáticos obtenidos para cada circuito electrónico, mediante los mapeos calculados, presentan características de forma y amplitud congruentes con los atractores caóticos típicos de los modelos matemáticos adimensionales de las figuras 9, 13, 17, 21, 25, 29 y 33. Por lo que se puede concluir que son válidas tanto las realizaciones electrónicas presentadas, los modelos matemáticos obtenidos (8), (13), (18), (23) y (28), así como los mapeos y equivalencias de parámetros y variables calculados para cada sistema caótico.

\section{CONCLUSIONES}

En este trabajo se muestra la realización y modelado de varios de los sistemas caóticos más conocidos con términos cuadráticos, utilizando multiplicadores y op-amp. Un circuito propuesto en este trabajo permite unificar la realización de los sistemas de Lorenz, Chen y Lü, con sólo ajustar algunos de sus componentes variables. El modelado y análisis realizados permite seleccionar con mayor libertad los componentes electrónicos de los circuitos para que su comportamiento sea consistente con el de los modelos matemáticos caóticos adimensionales 
establecidos en la literatura. Particularmente, con los modelos y equivalencias obtenidos fue posible ajustar los valores de componentes para acotar los atractores caóticos a rangos de operación admisibles en circuitos electrónicos. En las siguientes partes de este trabajo, se mostrará la realización electrónica de sistemas lineales por tramos y la implementación en dispositivos digitales.

\section{REFERENCIAS}

1. Lorenz, E.N. Deterministic Nonperiodic Flow. Journal of the Atmospheric Sciences, (1963) 20: 130-141.

2. Kevin M. Cuomo and Alan V. Oppenheim. Circuit implementation of Synchronized Chaos with Applications to Communications. Physical Review Letters. (1993) 71(65).

3. Olivares, D. Aplicación de sistemas caóticos en control automático. Tesis, Universidad Autónoma de Nuevo León, San Nicolás de los Garza, NL, México. (1994).

4. Pecora, L. M., Carroll, T. L., Johnson, G. A., Mar, D. J., and Heagy, J. F. Fundamentals of synchronization in chaotic systems, concepts, and applications. Chaos: An Interdisciplinary Journal of Nonlinear Science. (1997) 7(4): 520-543.

5. Jaimes-Reátegui, R. et. al. Secure optoelectronic communication using laser diode by chaotic Rössler circuits. Journal of Physics: Conference Series. (2001) 274: 012024.

6. García-Lopez, J. H. et. al. Novel communication scheme based on chaotic Rössler circuits. Journal of Physics: Conference Series. (2005) 23: 029

7. García-López, J. H. et. al. Synchronization of chaotic systems with coexisting attractors. Physical Review Letters. (2006) 96(24): 244102.

8. Rodriguez-Liñan, A., de León Morales, J. Sincronización de caos mediante observadores para cifrado en comunicaciones. Ingenierías. (2007) 10(34): 44-50.

9. Rössler, O. E. An equation for continuous chaos. Physics Letters A. (1976) 57(5): 397-398.

10. Braun, T. and Heisler, I. A. A comparative investigation of controlling chaos in a Rössler system. Physica A: Statistical Mechanics and its Applications. (2000) 283: 136-19.

11. L. Pecora, T. Caroll, \& J. Heagy. Synchronization in Chaotic Systems, Concepts and Applications. (2006).

12. Chiu, R., Mora-González, M., and Lopez-Mancilla, D. Osciladores Caóticos implementados en Microcontrolador PIC18F. Nova scientia. (2013) 6(12): 60-77.

13. P. Salas C. Análisis y diseño de sistemas caóticos clásicos con base en filtros pasa-bajas. Tesis, Universidad Autónoma de San Luis Potosí, San Luis Potosí, México (2018).

14. Wang, M.J. Chaotic Control of Lü System via Three Methods. International Journal of Modern Nonlinear Theory and Application, (2014) 3: 29-36.

15. Malasoma, J.M. A new class of minimal chaotic flows. Physics Letters A, 2002; 305: 52-58.

16. Hambley, A.R., Electronics, (2000) Upper Saddle River, N.J., Prentice Hall.

17. Lü, J. and Chen, G. A New Chaotic Attractor Coined. International Journal of Bifurcation and Chaos, (2002) $12,659-661$.

18. Chen, G. and Ueta, T. Yet Another Chaotic Attractor. International Journal of Bifurcation and Chaos, (1999) 9, 1465-1466.

19. Pehlivan İ, Kurt E, Lai Q, Basaran A, Kutlu M. A Multiscroll Chaotic Attractor and its Electronic Circuit Implementation, Chaos Theory and Applications. 2019; 1(1): 29-37.

20. Méndez-Ramírez, R., Cruz-Hernández, C., Arellano-Delgado, A. and Martínez-Clark, R. A new simple chaotic Lorenz-type system and its digital realization using a TFT touch-screen display embedded system. Complexity, 2017(6820492): 1-13. 